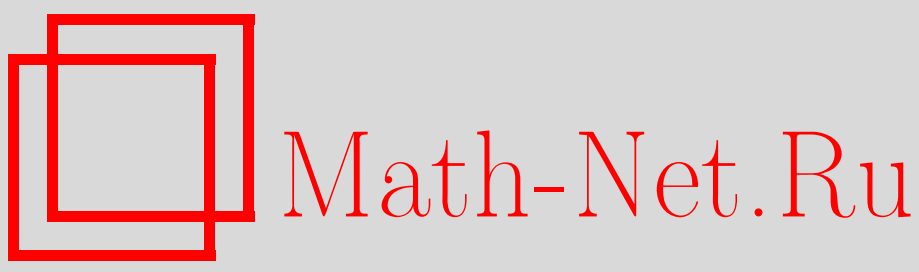

В. Ф. Кириченко, А. Р. Рустанов, Дифференциальная геометрия квази-сасакиевых многообразий, Матем. сб., 2002, том 193, номер 8, 71-100

DOI: https://doi.org/10.4213/sm675

Использование Общероссийского математического портала Math-Net.Ru подразумевает, что вы прочитали и согласны с пользовательским соглашением http: //www. mathnet.ru/rus/agreement

Параметры загрузки:

IP: 35.173 .219 .149

26 апреля 2023 г., $16: 26: 43$ 
УДК 514.76

\author{
В.Ф. Кириченко, А.Р. Рустанов
}

\title{
Дифференциальная геометрия квази-сасакиевых многообразий
}

\begin{abstract}
Получена полная группа структурных уравнений квази-сасакиевой структуры. На их основе изучено строение основных тензоров квази-сасакиева многообразия: тензора Римана-Кристоффеля, тензора Риччи и т.п. Получены интересные характеристики квази-сасакиевых многообразий Эйнштейна. Установлены дополнительные свойства симметрии тензора Римана-Кристоффеля. С их помощью выделен новый класс - класс $C R_{1}$ квази-сасакиевых многообразий. Приведено исчерпывающее описание локального строения многообразий этого класса. Получена полная классификация (с точностью до $\mathscr{B}$-преобразования метрики) многообразий этого класса, обладающих дополнительными свойствами типа изотропности.

Библиография: 19 названий.
\end{abstract}

Контактные и почти контактные структуры составляют один из наиболее содержательных примеров дифференциально-геометрических структур. Их теория является естественным обобшением так называемой контактной геометрии, имеющей существенные приложения в классической и квантовой механике. Их изучение именно как дифференциально-геометрических структур началось с появлением основополагающих работ Чженя [1], Дж. Грея [2], Сасаки [3]. Почти контактные метрические структуры являются нечетномерным аналогом почти эрмитовых структур, и между этими классами структур существует ряд важных взаимосвязей. Например, почти контактная метрическая структура внутренним образом возникает на гиперповерхности почти эрмитова многообразия, на пространстве главного $T^{1}$-расслоения над почти эрмитовым многообразием и т. д. Однако несмотря на это обстоятельство геометрия почти контактных метрических структур резко отличается от геометрии почти эрмитовых структур и требует при классическом подходе принципиально новых средств изучения [4]. Тем не менее, как показано первым из авторов, обе теории укладьваются в общие рамки так называемых обобшенных почти эрмитовых структур, и такой подход позволил получить сушественные продвижения в обоих направлениях [5]-[8].

Одним из главных аспектов аппарата обобшенной эрмитовой геометрии является метод присоединенных $G$-структур, широко используемый в настояшей работе. Эта работа посвящена изучению геометрии обширного класса почти контактных метрических многообразий келерова типа, известного под названием $к \boldsymbol{\varepsilon} a-$ зи-сасакиевых (quasi-Sasakian [9]) многообразий. Квази-сасакиевы многообразия интересны тем, что они заполняют естественную нишу между двумя контактньми аналогами келеровых многообразий, а именно сасакиевыми и косимплектическими

(C) В.Ф. КИРИченко, А.Р. РУСтАнов 2002 
многообразиями. Квази-сасакиевы многообразия введены в работах Блэра. После своей диссертационной работы (1966 г.) им была опубликована статья [9], заложившая основы теории квази-сасакиевых многообразий. Впоследствии появились работы Танно [10], Канемаки [11], Янамото [12] и других авторов, в которых квази-сасакиевы многообразия рассматривались с различных точек зрения. Одно из наиболее популярных направлений изучения квази-сасакиевых многообразий заключено в исследовании их локального строения. Именно хорошо известно, что произведение многообразия Сасаки на келерово многообразие является квази-сасакиевым многообразием [13]. Возникает вопрос: когда квази-сасакиево многообразие имеет такое строение хотя бы локально? На этот счет Блэром [9] и Канемаки [11] получены некоторые достаточные условия. В нашей работе дан исчерпывающий ответ на этот вопрос в терминах дополнительных свойств симметрии тензора римановой кривизны квази-сасакиева многообразия. При этом естественно выделился так называемый класс $C R_{1}$ квази-сасакиевых многообразий, представляющий существенный интерес с различных точек зрения. Этот результат достигнут систематическим использованием полной группы структурных уравнений присоединенной $G$-структуры, полученной в настояшей работе, позволившим досконально изучить дополнительные свойства симметрии тензора римановой кривизны квази-сасакиева многообразия, выделить несколько интересных классов квази-сасакиевых многообразий и изучить их геометрические характеристики. Наиболее подробно исследованы квази-сасакиевы многообразия класса $C R_{1}$. Получено в определенном смысле исчерпывающее описание локального строения многообразий этого класса. Приведена полная классификация (с точностью до $\mathscr{B}$-преобразования метрики) квази-сасакиевых многообразий класса $C R_{1}$ постоянной $\Phi$-голоморфной секционной кривизны, а также квази-сасакиевых многообразий этого класса, удовлетворяющих аксиоме $\Phi$-голоморфных $(2 r+1)$-плоскостей, что существенно обобщает известные результаты Танно, касающиеся классификации сасакиевых пространственных форм [14], а также обобщает и углубляет результаты Огиуэ [15] и Исихары [16], касающиеся изучения почти контактных метрических многообразий, в частности многообразий Сасаки, удовлетворяющих аксиоме $\Phi$-голоморфных $(2 r+1)$-плоскостей.

Пусть $M$ - гладкое многообразие, $\mathfrak{X}(M)-C^{\infty}(M)$-модуль гладких векторных полей на $M, d$ - оператор внешнего дифференцирования. Все многообразия, тензорные поля и подобные объекты предполагаются гладкими класса $C^{\infty}$. Напомним [4], что почти контактной метрической структурой на $M$ называется совокупность $(\Phi, \xi, \eta, g=\langle\cdot, \cdot\rangle)$ тензорных полей на $M$, где $\Phi$ - тензор типа $(1,1)$, называемый структурнылм әндоморфизмом, $\xi$ и $\eta$ - вектор и ковектор, называемые соответственно структурным вектором и контактной формой, $g$ - (псевдо)риманова метрика. При этом

$$
\begin{gathered}
\eta(\xi)=1, \quad \Phi(\xi)=0, \quad \eta \circ \Phi=0, \\
\Phi^{2}=-\mathrm{id}+\eta \otimes \xi, \quad\langle\Phi X, \Phi Y\rangle=\langle X, Y\rangle-\eta(X) \eta(Y),
\end{gathered}
$$

$X, Y \in \mathfrak{X}(M)$. Легко проверить, что тензор $\Omega(X, Y)=\langle X, \Phi Y\rangle$ кососимметричен, т.е. является 2-формой на $M$. Он называется фундаментальной формой струкmурь. Хорошо известно, что необходимьпи условиями существования почти контактной метрической структуры на многообразии является его нечетномерность и 
ориентируемость. Многообразие, на котором фиксирована почти контактная метрическая структура, называется почти контактным метрическим многообразием. Два почти контактных метрических многообразия $M_{1}$ и $M_{2}$ называются голоморфно изометричными, или әквивалентными, если существует изометрический диффеоморфизм $f: M_{1} \rightarrow M_{2}$ такой, что $\Phi_{2} \circ f_{*}=f_{*} \circ \Phi_{1}$, где $\Phi_{1}$ и $\Phi_{2}-$ структурные эндоморфизмы многообразий $M_{1}$ и $M_{2}$ соответственно, $f_{*}$ - отображение увлечения векторных полей, порожденное диффеоморфизмом $f$.

В случае, когда $d \eta=\Omega$, почти контактная метрическая структура называется контактной метрической структурой.

Почти контактная метрическая структура называется нормальной, если

$$
N+2 d \eta \otimes \xi=0
$$

где $N$ - тензор Нейенхейса оператора $\Phi$. Нормальная контактная метрическая структура называется сасакиевой структурой. Классическим примером сасакиевой структуры является почти контактная метрическая структура на нечетномерной сфере $S^{2 n+1}$, канонически вложенной в комплексное евклидово пространство $\mathbb{C}^{n}$.

ОПРЕДЕЛЕНИЕ 1. Почти контактная метрическая структура называется $\boldsymbol{\kappa} \boldsymbol{\boldsymbol { \theta }} \boldsymbol{a -}$ зи-сасакиевой (короче, $Q \mathscr{S}$-) структурой, если она нормальна, а ее фундаментальная форма замкнута.

Очевидно, всякая сасакиева структура является $Q \mathscr{S}$-структурой. Другим важньг примером $Q \mathscr{S}$-структуры является косимплектическая структура, которую можно охарактеризовать как нормальную контактную метрическую структуру с замкнутыми контактной и фундаментальной формами [11].

Пусть $(\Phi, \xi, \eta, g)$ - почти контактная метрическая структура на многообразии $M^{2 n+1}$. В модуле $\mathfrak{X}(M)$ внутренним образом определены два взаимно дополнительных проектора $\mathbf{m}=\eta \otimes \xi$ и $\mathbf{l}=\mathrm{id}-\mathbf{m}=-\Phi^{2}$; таким образом, $\mathfrak{X}(M)=\mathscr{L} \oplus \mathscr{M}$, где $\mathscr{M}=\operatorname{Im} \mathbf{m}-$ линейная оболочка структурного вектора, $\mathscr{L}=\operatorname{Im} \Phi=\operatorname{ker} \eta-$ так называемое контактное распределение. Очевидно, распределения $\mathscr{M}$ и $\mathscr{L}$ инвариантны относительно $\Phi$ и взаимно ортогональны. Очевидно также, что

$$
\widetilde{\Phi}^{2}=-\mathrm{id}, \quad\langle\widetilde{\Phi} X, \widetilde{\Phi} Y\rangle=\langle X, Y\rangle, \quad X, Y \in \mathscr{L},
$$

где $\widetilde{\Phi}=\left.\Phi\right|_{\mathscr{L}}$. Поэтому если $p \in M$, то в касательном пространстве $T_{p}(M)$ можно построить ортонормированный репер $\left(p, e_{0}, e_{1}, \ldots, e_{n}, \Phi e_{1}, \ldots, \Phi e_{n}\right)$, где $e_{0}=\xi_{p}$. Такой репер будем назьвать вещественно адаптированным репером. $\mathrm{C}$ другой стороны, пусть $\mathscr{L}^{c}=\mathscr{L} \otimes \mathbb{C}$ - комплексификация распределения $\mathscr{L}$. В ней внутренним образом определены два взаимно дополнительных проектора $\sigma=\frac{1}{2}(\mathrm{id}-\sqrt{-1} \Phi)$ и $\bar{\sigma}=\frac{1}{2}(\mathrm{id}+\sqrt{-1} \Phi)$ на собственные подмодули $\mathscr{D}_{\Phi}^{\imath}$ и $\mathscr{D}_{\Phi}^{-\imath}$ эндоморфизма $\Phi$, отвечающие собственным значениям $\sqrt{-1}$ и $-\sqrt{-1}$ соответственно. Следовательно, можно построить репер $\left(p, \varepsilon_{0}, \varepsilon_{1}, \ldots, \varepsilon_{n}, \varepsilon_{\hat{1}}, \ldots, \varepsilon_{\widehat{n}}\right)$ комплексификации пространства $T_{p}(M)$, где $\varepsilon_{0}=\xi_{p}, \varepsilon_{a}=\sqrt{2} \sigma\left(e_{a}\right), \varepsilon_{\widehat{a}}=\sqrt{2} \bar{\sigma}\left(e_{a}\right)$, состоящий 
из собственных векторов оператора $\Phi_{p}$. Такой репер называется $A$-репером. Легко видеть, что матрицы компонент тензоров $\Phi_{p}$ и $g_{p}$ в $A$-репере имеют вид соответственно

$$
\left(\Phi_{j}^{i}\right)=\left(\begin{array}{ccc}
0 & 0 & 0 \\
0 & \sqrt{-1} I_{n} & 0 \\
0 & 0 & -\sqrt{-1} I_{n}
\end{array}\right), \quad\left(g_{i j}\right)=\left(\begin{array}{ccc}
1 & 0 & 0 \\
0 & 0 & I_{n} \\
0 & I_{n} & 0
\end{array}\right)
$$

где $I_{n}$ - единичная матрица порядка $n$. Хорошо известно [5], [6], что совокупность таких реперов определяет $G$-структуру на $M$ со структурной группой $\{1\} \times U(n)$, представленной матрицами вида $\left(\begin{array}{ccc}1 & 0 & 0 \\ 0 & A & 0 \\ 0 & 0 & \bar{A}\end{array}\right)$, где $A \in U(n)$. Эта $G$-структура называется присоединенной. Еще раз подчеркнем, что пространство присоединенной $G$-структуры состоит из комплексных реперов, т.е. реперов комплексификации соответствующих касательных пространств. Поэтому, даже имея дело с вещественными тензорами, мы, говоря об их компонентах на пространстве присоединенной $G$-структуры, подразумеваем компоненты комплексных расшпрений этих тензоров. В свою очередь, комплексный тензор является комплексным расширением вещественного тензора тогда и только тогда, когда он инвариантен относительно оператора комплексного сопряжения. Следуя общепринятой традиции, будем называть такой тензор вешественным. В частности, сумма чистого комплексного тензора и комплексно сопряженного ему тензора является вещественным тензором.

На протяжении всей работы будем подразумевать, что индексы $i, j, k, \ldots$ пробегают значения от 0 до $2 n$, индексы $a, b, c, d, f, g, \ldots$ - значения от 1 до $n$, и положим $\widehat{a}=a+n, \hat{\hat{a}}=a, \hat{0}=0$. Поскольку $\Phi$ и $g$ - тензоры типов $(1,1)$ и $(2,0)$ соответственно, их компоненты на пространстве расслоения всех комплексных реперов над $M$ удовлетворяют уравнениям

$$
\begin{aligned}
d \Phi_{j}^{i}+\Phi_{k}^{i} \omega_{j}^{k}-\Phi_{j}^{k} \omega_{k}^{i} & =\Phi_{j, k}^{i} \omega^{k} \\
d g_{i j}+g_{k j} \omega_{i}^{k}+g_{i k} \omega_{j}^{k} & =g_{i j, k} \omega^{k}
\end{aligned}
$$

где $\left\{\omega^{i}\right\},\left\{\omega_{j}^{i}\right\}$ - компоненты форм смещения и римановой связности $\nabla$ соответственно, $\Phi_{j, k}^{i}, g_{i j, k}$ - компоненты ковариантного диффференциала тензоров $\Phi$ и $g$ в этой связности соответственно. Более того, в силу определения римановой связности $\nabla g=0$ и, значит,

$$
g_{i j, k}=0 \text {. }
$$

С учетом (1) и (3) соотношения (2) на пространстве присоединенной $G$-структуры перепишутся в форме

$$
\begin{array}{ll}
\Phi_{b, k}^{a}=0, \quad \Phi_{\widehat{b}, k}^{\widehat{a}}=0, & \Phi_{0, k}^{0}=0, \\
\omega_{\widehat{b}}^{a}=-\frac{1}{2} \sqrt{-1} \Phi_{\widehat{b}, k}^{a} \omega^{k}, & \omega_{b}^{\widehat{a}}=\frac{1}{2} \sqrt{-1} \Phi_{b, k}^{\widehat{a}} \omega^{k}, \\
\omega_{0}^{a}=-\sqrt{-1} \Phi_{0, k}^{a} \omega^{k}, & \omega_{0}^{\widehat{a}}=\sqrt{-1} \Phi_{0, k}^{\widehat{a}} \omega^{k}, \\
\omega_{b}^{0}=\sqrt{-1} \Phi_{b, k}^{0} \omega^{k}, & \omega_{\widehat{b}}^{0}=-\sqrt{-1} \Phi_{\widehat{b}, k}^{0} \omega^{k}, \\
\omega_{0}^{0}=0, & \omega_{j}^{i}+\omega_{\hat{\imath}}^{\hat{\jmath}}=0 .
\end{array}
$$


Кроме того, заметим, что в силу вещественности соответствующих форм и тензоров $\bar{\omega}^{i}=\omega^{\hat{\imath}}, \bar{\omega}_{j}^{i}=\omega_{\hat{\jmath}}^{\hat{\imath}}, \overline{\nabla \Phi}_{j, k}^{i}=\nabla \Phi_{\hat{\jmath}, \widehat{k}}^{\hat{\imath}}$, где $t \rightarrow \bar{t}$ - оператор комплексного сопряжения.

С учетом этих соотношений первая группа структурных уравнений римановой связности

$$
d \omega^{i}=\omega_{j}^{i} \wedge \omega^{j}
$$

на пространстве присоединенной $G$-структуры почти контактного метрического многообразия запишется в следующей форме, называемой первой группой структурных уравнений почти контактного метрического многообразия:

$$
\begin{aligned}
d \omega^{a} & =\omega_{b}^{a} \wedge \omega^{b}+B^{a b}{ }_{c} \omega^{c} \wedge \omega_{b}+B^{a b c} \omega_{b} \wedge \omega_{c}+B^{a b} \omega \wedge \omega_{b}+B^{a}{ }_{b} \omega \wedge \omega^{b}, \\
d \omega_{a} & =-\omega_{a}^{b} \wedge \omega_{b}+B_{a b}{ }^{c} \omega_{c} \wedge \omega^{b}+B_{a b c} \omega^{b} \wedge \omega^{c}+B_{a b} \omega \wedge \omega^{b}+B_{a}{ }^{b} \omega \wedge \omega_{b}, \\
d \omega & =C_{a b} \omega^{a} \wedge \omega^{b}+C^{a b} \omega_{a} \wedge \omega_{b}+C_{b}^{a} \omega^{b} \wedge \omega_{a}+C_{a} \omega \wedge \omega^{a}+C^{a} \omega \wedge \omega_{a},
\end{aligned}
$$

где

$$
\begin{array}{rlrl}
\omega & =\omega^{0}, & \omega_{i} & =g_{i j} \omega^{j}, \\
B^{a b c} & =\frac{\sqrt{-1}}{2} \Phi_{[\widehat{b}, \widehat{c}]}^{a}, & B_{a b c} & =-\frac{\sqrt{-1}}{2} \Phi_{[b, c]}^{\widehat{a}}, \\
B^{a b}{ }_{c} & =-\frac{\sqrt{-1}}{2} \Phi_{\widehat{b}, c}^{a}, & B_{a b}{ }^{c} & =\frac{\sqrt{-1}}{2} \Phi^{\widehat{a}}{ }_{b, \widehat{c}}, \\
B^{a b} & =-\sqrt{-1}\left(\frac{1}{2} \Phi_{\widehat{b}, 0}^{a}-\Phi_{0, \widehat{b}}^{a}\right), & B_{a b} & =\sqrt{-1}\left(\frac{1}{2} \Phi_{b, 0}^{\widehat{a}}{ }_{b, 0} \Phi_{0, b}^{\widehat{a}}\right), \\
B^{a}{ }_{b} & =\sqrt{-1} \Phi_{0, b}^{a}, \\
C^{a b} & =\sqrt{-1} \Phi_{[\widehat{a}, \widehat{b}]}^{0}, & B_{a}{ }^{b} & =-\sqrt{-1} \Phi_{0, \widehat{a}}^{\widehat{a}}, \\
C_{b}^{a} & =-\sqrt{-1}\left(\Phi_{b, \widehat{a}}^{0}+\Phi_{\widehat{a}, b}^{0}\right), & C_{a b} & =-\sqrt{-1} \Phi_{[a, b]}^{0}, \\
C^{a} & =-\sqrt{-1} \Phi_{\widehat{a}, 0}^{0}, \quad C_{a}=\sqrt{-1} \Phi_{a, 0}^{0} .
\end{array}
$$

При этом

$$
\overline{B^{a b c}}=B_{a b c}, \quad \overline{B^{a b}}=B_{a b}, \quad \overline{\omega_{b}^{a}}=-\omega_{a}^{b} .
$$

Напомним [17], что тензором (или оператором) Нейенхейса эндоморфизма $\Phi$ касательного пучка многообразия называется тензор $N$ типа $(2,1)$, определяемый формулой

$$
N(X, Y)=\frac{1}{4}\left(\Phi^{2}[X, Y]+[\Phi X, \Phi Y]-\Phi[\Phi X, Y]-\Phi[X, \Phi Y]\right), \quad X, Y \in \mathfrak{X}(M)
$$

Его обращение в нуль равносильно интегрируемости структуры [17]. Прямой подсчет с учетом тождества $[X, Y]=\nabla_{X} Y-\nabla_{Y} X$ показывает, что

$$
N(X, Y)=\frac{1}{4}\left(\nabla_{\Phi X}(\Phi) Y-\Phi \nabla_{X}(\Phi) Y-\nabla_{\Phi Y}(\Phi) X+\Phi \nabla_{Y}(\Phi) X\right)
$$


С учетом (4) получаем отсюда, что на пространстве присоединенной $G$-структуры компоненты тензора $N$ определяются тождествами

$$
\begin{gathered}
N_{a b}^{0}=-\frac{\sqrt{-1}}{2} \Phi_{0[a, b]}, \quad N_{\widehat{a} b}^{0}=-N_{b \widehat{a}}^{0}=-\frac{\sqrt{-1}}{2} \Phi_{(\widehat{a}, b)}^{0}, \quad N_{\widehat{a} \widehat{b}}^{0}=\frac{\sqrt{-1}}{2} \Phi^{0[a, b]}, \\
N_{\widehat{b} 0}^{a}=-N_{0 \widehat{b}}^{a}=\frac{\sqrt{-1}}{4} \Phi^{a b, 0}-\frac{\sqrt{-1}}{2} \Phi^{a 0, b}, \quad N_{\widehat{b} \widehat{c}}^{a}=\sqrt{-1} \Phi^{a[b, c]}, \\
N_{b c}^{\widehat{a}}=-\sqrt{-1} \Phi_{a[b, c]}, \quad N_{b 0}^{\widehat{a}}=-N_{0 b}^{\widehat{a}}=\frac{\sqrt{-1}}{2} \Phi_{a 0, b}-\frac{\sqrt{-1}}{4} \Phi_{a b, 0} .
\end{gathered}
$$

Остальные компоненты тензора Нейенхейса тождественно равны нулю.

С другой стороны, учитывая, что $\omega=\pi^{*}(\eta)$, где $\pi$ - естественная проекция пространства присоединенной $G$-структуры на многообразие $M$, а также то обстоятельство, что на пространстве присоединенной $G$-структуры $\xi^{a}=\xi^{\widehat{a}}=0, \xi^{0}=1$, с учетом (5) находим, что на этом пространстве

$$
\begin{aligned}
& (\xi \otimes d \eta)_{i j}^{a}=(\xi \otimes d \eta)_{i j}^{\widehat{a}}=0 \\
& (\xi \otimes d \eta)_{b c}^{0}=-\sqrt{-1} \Phi_{[b, c]}^{0}, \\
& (\xi \otimes d \eta)_{b \widehat{c}}^{0}=-(\xi \otimes d \eta)_{\widehat{c} b}^{0}=-\sqrt{-1} \Phi_{(b, \widehat{c})}, \\
& (\xi \otimes d \eta)_{a 0}^{0}=-(\xi \otimes d \eta)_{0 a}^{0}=-\frac{\sqrt{-1}}{2} \Phi_{a, 0}^{0} \\
& (\xi \otimes d \eta)_{\widehat{a} 0}^{0}=-(\xi \otimes d \eta)_{0 \widehat{a}}^{0}=\frac{\sqrt{-1}}{2} \Phi_{\widehat{a}, 0}^{0}, \\
& (\xi \otimes d \eta)_{00}^{0}=0
\end{aligned}
$$

С учетом (9) и (10) несложно подсчитать, что почти контактная метрическая структура нормальна тогда и только тогда, когда на пространстве присоединенной G-структуры

$$
\Phi_{b, c}^{\widehat{a}}=\Phi_{\widehat{b}, \widehat{c}}^{a}=\Phi_{b, 0}^{\widehat{a}}=\Phi_{\widehat{b}, 0}^{a}=\Phi_{a, b}^{0}=\Phi_{\widehat{a}, \widehat{b}}^{0}=\Phi_{a, 0}^{0}=\Phi_{\widehat{a}, 0}^{0}=0,
$$

в силу чего первая группа (5) структурных уравнений нормальной структуры примет вид:

$$
\begin{aligned}
d \omega & =C_{b}^{a} \omega^{b} \wedge \omega_{a}, \\
d \omega^{a} & =\omega_{b}^{a} \wedge \omega^{b}+B^{a b}{ }_{c} \omega^{c} \wedge \omega_{b}+B^{a}{ }_{b} \omega \wedge \omega^{b}, \\
d \omega_{a} & =-\omega_{a}^{b} \wedge \omega_{b}+B_{a b}{ }^{c} \omega_{c} \wedge \omega^{b}+B_{a}{ }^{b} \omega \wedge \omega_{b} .
\end{aligned}
$$

Заметим, что соотношения (11) с учетом (4) можно переписать в форме

$$
\Phi_{a, 0}^{i}=0, \quad \Phi_{b, c}^{i}=0 .
$$

Рассмотрим первое из этих соотношений. В фиксированной точке $p \in M$ оно, очевидно, равносильно соотношению $\nabla_{\varepsilon_{0}}(\Phi)\left(\varepsilon_{a}\right)=0$. Поскольку $\xi_{p}=\varepsilon_{0}$, а векторы $\left\{\varepsilon_{a}\right\}$ образуют базис подпространства $\left(\mathscr{D}_{\Phi}^{2}\right)_{p}$, это соотношение равносильно тождеству

$$
\nabla_{\xi}(\Phi) \widetilde{X}=0, \quad \widetilde{X} \in \mathscr{D}_{\Phi}^{\imath} .
$$


Учитывая, что эндоморфизм $\sigma=\frac{1}{2}(\mathrm{id}-\sqrt{-1} \Phi)$ является проектором модуля $\mathscr{L}^{c}$ на подмодуль $\mathscr{D}_{\Phi}^{\imath}$, тождество (14) можно переписать в форме

$$
\nabla_{\xi}(\Phi)(\mathrm{id}-\sqrt{-1} \Phi) X=0, \quad X \in \mathscr{L} .
$$

Раскрывая это соотношение по линейности и выделяя действительную и мнимую части полученного равенства, приходим к эквивалентному тождеству:

$$
\nabla_{\xi}(\Phi) X=0, \quad X \in \mathscr{L} .
$$

Наконец, учитывая, что $\mathscr{L}=\operatorname{Im} \Phi$, получаем, что последнее тождество равносильно тождеству

$$
\nabla_{\xi}(\Phi) \Phi X=0, \quad X \in \mathfrak{X}(M) .
$$

Назовем описанную процедуру процедурой восстановления тожсдетва.

Аналогично, проводя процедуру восстановления по второму из соотношений (13), получаем, что оно равносильно тождеству

$$
\nabla_{\Phi X}(\Phi) \Phi Y-\nabla_{\Phi^{2} X}(\Phi) \Phi^{2} Y=0, \quad X, Y \in \mathfrak{X}(M) .
$$

Заметим, что тождества $(*)$ и $(* *)$ равносильны тождеству

$$
\nabla_{X}(\Phi) \Phi Y-\nabla_{\Phi X}(\Phi) \Phi^{2} Y=0 .
$$

В самом деле, заменив в (15) $X$ на $\xi$ (соответственно на $\Phi X$ ), мы получим тождества $(*)$ и $(* *)$ соответственно. С другой стороны, пусть справедливы тождества $(*)$ и $(* *)$, и пусть $X \in \mathfrak{X}(M)$. Тогда $X=\lambda \xi+\Phi Z$ для некоторых $\lambda \in C^{\infty}(M)$ и $Z \in \mathfrak{X}(M)$. С учетом этого

$$
\begin{aligned}
\nabla_{X}(\Phi) \Phi Y & =\lambda \nabla_{\xi}(\Phi) \Phi Y+\nabla_{\Phi Z}(\Phi) \Phi Y=\nabla_{\Phi Z}(\Phi) \Phi Y \\
& =\nabla_{\Phi^{2} Z}(\Phi) \Phi^{2} Y=\nabla_{\Phi X}(\Phi) \Phi^{2} Y .
\end{aligned}
$$

Тем самым установлен удобный аналитический критерий нормальности почти контактного метрического многообразия:

ПРЕДЛОЖЕНИЕ 1. Почти контактное метрическое многообразие нормально тогда и только тогда, когда на нем выполняется тожсдество

$$
\nabla_{X}(\Phi) \Phi Y-\nabla_{\Phi X}(\Phi) \Phi^{2} Y=0, \quad X, Y \in \mathfrak{X}(M) .
$$

Далее, компоненты фундаментальной формы структуры на пространстве присоединенной $G$-структуры имеют вид $\Omega_{a \widehat{b}}=-\Omega_{\widehat{b} a}=-\sqrt{-1} \delta_{a}^{b}$, а значит,

$$
\Omega=-2 \sqrt{-1} \omega^{a} \wedge \omega_{a}
$$

Дифференцируя это соотношение внешним образом с учетом (12), убеждаемся, что эта форма на нормальном многообразии замкнута тогда и только тогда, когда

$$
\Phi_{\widehat{b}, c}^{a}=\Phi_{b, \widehat{c}}^{\widehat{a}}=0, \quad \Phi_{0, \widehat{b}}^{\widehat{a}}=\Phi_{0, a}^{b},
$$

откуда с учетом (6) и (11) находим, что

$$
B^{a b}{ }_{c}=B_{a b}{ }^{c}=0, \quad C_{b}^{a}=2 B^{a}{ }_{b}=-2 B_{b}{ }^{a} .
$$

Следовательно, первая группа структурных уравнений $Q \mathscr{S}$-структуры имеет вид:

$$
\begin{aligned}
d \omega & =2 B_{b}^{a} \omega^{b} \wedge \omega_{a}, \\
d \omega^{a} & =\omega_{b}^{a} \wedge \omega^{b}+B_{b}^{a} \omega \wedge \omega^{b}, \\
d \omega_{a} & =-\omega_{a}^{b} \wedge \omega_{b}-B_{a}^{b} \omega \wedge \omega_{b},
\end{aligned}
$$

где $B_{b}^{a}=B_{b}^{a}=-B_{b}{ }^{a}=\sqrt{-1} \Phi_{0, b}^{a}$. 
Замечание 1 . Очевидно, $\overline{B_{b}^{a}}=-B_{a}^{b}$.

Далее, с учетом (16) и (18) условие $d \eta=\Omega($ соответственно $d \eta=0)$ на пространстве присоединенной $G$-структуры $Q \mathscr{S}$-многообразия задается соотношениями $B_{b}^{a}=-\sqrt{-1} \delta_{b}^{a}$ (соответственно $\left.B_{b}^{a}=0\right)$. Получено

ПРЕДЛОЖЕНИЕ 2. QS -структура является сасакиевой (соответственно косимплектической) тогда и только тогда, когда на пространстве присоединенной G-структурь

$$
B_{b}^{a}=-\sqrt{-1} \delta_{b}^{a} \quad\left(\text { соответственно } B_{b}^{a}=0\right) .
$$

Стандартная процедура дифференциального продолжения соотношений (17) позволяет получить вторую группу структурных уравнений $Q \mathscr{S}$-структуры:

$$
d \omega_{b}^{a}=\omega_{c}^{a} \wedge \omega_{b}^{c}+\left(A_{b c}^{a d}-2 B_{b}^{a} B_{c}^{d}\right) \omega^{c} \wedge \omega_{d}+B_{b c}^{a} \omega \wedge \omega^{c}+B_{d}^{a c} \omega \wedge \omega^{c},
$$

где $\left\{A_{b c}^{a d}\right\}$ - семейство функций на пространстве присоединенной $G$-структуры, служащих компонентами так называемого тензора кривизны присоединенной $Q$-алгебрьг [5], или структурного тензора второго рода, причем

$$
A_{[b c]}^{a d}=0, \quad A_{b c}^{[a d]}=0, \quad \overline{A_{b c}^{a d}}=A_{a d}^{b c}
$$

Кроме того,

$$
d B_{b}^{a}-B_{b}^{h} \omega_{h}^{a}+B_{h}^{a} \omega_{b}^{h}=B_{b c}^{a} \omega^{c}+B_{b}^{a c} \omega_{c}
$$

где $B_{b c}^{a}, B_{b}^{a c}$ - подходящие функции на пространстве присоединенной $G$-структуры, причем

$$
\begin{aligned}
B_{[b c]}^{a} & =B_{b}^{[a c]}=0, \\
\overline{B_{b c}^{a}} & =-B_{a}^{b c} .
\end{aligned}
$$

Из (21) следует, что набор функций $\left\{B_{b}^{a}\right\}$ является набором компонент (комплексного) тензора $B$ типа $(1,1)$ на $M$, который мы назовем структурным тензором первого рода. В силу замечания 1 этот тензор является косоэрмитовым оператором в модуле $\mathscr{D}_{\Phi}^{\imath}$ относительно стандартной эрмитовой метрики [5].

Сравнивая соотношения (19) со второй группой структурных уравнений римановой связности

$$
d \omega_{j}^{i}=\omega_{k}^{i} \wedge \omega_{j}^{k}+\frac{1}{2} R_{j k l}^{i} \omega^{k} \wedge \omega^{l},
$$

где $R_{j k l}^{i}$ - компоненты тензора Римана-Кристоффеля, в силу линейной независимости базисных форм получаем, что на пространстве присоединенной $G$-структуры

$$
\begin{aligned}
& R_{\widehat{a} b c \widehat{d}}=A_{b c}^{a d}-2 B_{b}^{a} B_{c}^{d}-B_{c}^{a} B_{b}^{d}, \\
& R_{\widehat{a} b 0 c}=B_{b c}^{a} \\
& R_{\widehat{a} b 0 \widehat{c}}=B_{b}^{a c} .
\end{aligned}
$$


Кроме того, с учетом (4), (6), (11) и (17) имеем:

$$
\begin{aligned}
& \omega^{a b} \equiv \omega_{\widehat{b}}^{a}=0, \quad \omega_{a b} \equiv \omega_{b}^{\widehat{a}}=0 \\
& \omega_{0}^{a}=-B_{c}^{a} \omega^{c}, \quad \omega_{a}^{0}=-B_{a}^{c} \omega_{c} .
\end{aligned}
$$

Дифференциальное продолжение этих соотношений с учетом (18), (21), (23) и (25) приводит к равенствам

$$
R_{\widehat{a} \widehat{b} c d}=2 B_{[c}^{a} B_{d]}^{b}, \quad R_{\widehat{a} 0 b 0}=B_{c}^{a} B_{b}^{c},
$$

а также к соотношениям, полученньм из (24) и (26) с учетом классических свойств симметрии и вешественности тензора Римана-Кристоффеля. Все остальные компоненты этого тензора равны нулю.

С использованием (24) и (26) вычислим компоненты $S_{i j}=R_{i j k}^{k}$ тензора Риччи $S$ на пространстве присоединенной $G$-структуры:

$$
\begin{aligned}
S_{a b} & =S_{\widehat{a} \widehat{b}}=0, \\
S_{\widehat{a} b} & =S_{b \widehat{a}}=2 B_{c}^{a} B_{b}^{c}-A_{b c}^{a c}, \\
S_{a 0} & =S_{0 a}=B_{a b}^{b}, \\
S_{\widehat{a} 0} & =S_{0 \widehat{a}}=B_{b}^{a b} \\
S_{00} & =-2 B_{b}^{a} B_{a}^{b} .
\end{aligned}
$$

В частности, скалярная кривизна $\varkappa Q \mathscr{S}$-многообразия вычисляется по формуле

$$
\varkappa=g^{i j} S_{i j}=2\left(B_{b}^{a} B_{a}^{b}-A_{a b}^{a b}\right) .
$$

Пусть, в частности, $M$ - многообразие Эйнштейна с космологической константой $\varepsilon$, т.е. $S_{i j}=\varepsilon g_{i j}$. Тогда $S_{\widehat{a} b}=\varepsilon \delta_{b}^{a}, S_{a 0}=0, S_{00}=\varepsilon$. С учетом $\left(27_{3}\right) B_{a c}^{c}=0$ и, кроме того, с учетом $\left(27_{2}\right)$ и $\left(27_{5}\right)$

$$
2 \operatorname{tr}\left(B^{2}\right)-A_{a c}^{a c}=\varepsilon n, \quad-2 \operatorname{tr}\left(B^{2}\right)=\varepsilon,
$$

откуда $A_{a c}^{a c}=2(n+1) \operatorname{tr}\left(B^{2}\right), \varkappa=-2(2 n+1) \operatorname{tr}\left(B^{2}\right)$,

$$
\varepsilon=-2 \operatorname{tr}\left(B^{2}\right)
$$

Заметим, что в силу косоэрмитовости оператора $B_{p}$ при подходящем выборе $A$-репера $\left\{p, \varepsilon_{a}, \varepsilon_{\widehat{b}}\right\}$ он приводится к диагональному виду с чисто мнимыми собственными значениями, т.е.

$$
B_{b}^{a}=B_{b} \delta_{b}^{a}, \quad B_{b}=\sqrt{-1} \alpha_{b}, \quad \alpha_{b} \in \mathbb{R}, \quad b=1, \ldots, n .
$$

В таком базисе $\operatorname{tr}\left(B^{2}\right)=-\sum_{b=1}^{n}\left(\alpha_{b}\right)^{2} \leqslant 0$. Из (29) следует, что $\varepsilon \geqslant 0$. Если $\varepsilon=0$, то $\operatorname{tr}\left(B^{2}\right)=0$, т.е. $0=B_{b}^{a} B_{a}^{b}=-\sum_{a, b=1}^{n}\left|B_{b}^{a}\right|^{2}$, а значит, $B_{b}^{a}=0$, т.е. $M-$ косимплектическое многообразие. Согласно [18] $M$ локально голоморфно изометрично многообразию вида $\mathbb{R} \times N^{2 n}$, где $N^{2 n}$ - риччи-плоское келерово многообразие. Если $\varepsilon>0$, то по классической теореме Майерса [17] в случае полноты $M$ компактно и имеет конечную фундаментальную группу. Мы получаем следующий результат: 


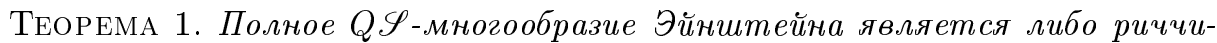
плоским косимплектическим многообразием, а значит, голоморфно изометрично накрывается произведением вещественной прямой на риччи-плоское келерово многообразие, либо компактно и имеет конечную фундаментальную группу.

Выясним геометрический смысл первого структурного тензора. Поскольку $\xi-$ тензор типа $(0,1)$, его компоненты на пространстве расслоения реперов многообразия $M$ удовлетворяют уравнениям

$$
d \xi^{i}-\xi^{j} \omega_{j}^{i}=\xi^{i}, j \omega^{j}
$$

где $\xi^{i},{ }_{j}$ - компоненты тензора $\mathscr{B}=\nabla \xi$. Расписывая эти соотношения на пространстве присоединенной $G$-структуры с учетом (25) и того обстоятельства, что на этом пространстве $\xi^{a}=0, \xi^{\widehat{b}}=0, \xi^{0}=1$, получаем, что

$$
\xi_{, b}^{a}=B_{b}^{a}, \quad \xi_{, \widehat{b}}^{a}=\xi_{, 0}^{a}=0, \quad \xi_{, a}^{0}=\xi_{, \widehat{a}}^{0}=\xi_{, 0}^{0}=0 .
$$

С учетом вещественности вектора $\xi$ и в силу замечания 1 этот результат можно сформулировать следуюшим образом:

ПРЕДЛОЖЕНИЕ 3. На пространстве присоединенной G-структуры матрица оператора $\mathscr{B}$ имеет вид:

$$
\left(\mathscr{B}_{j}^{i}\right) \equiv\left(\xi^{i},{ }_{j}\right)=\left(\begin{array}{ccc}
0 & 0 & 0 \\
0 & \left(B_{b}^{a}\right) & 0 \\
0 & 0 & -\left(B_{b}^{a}\right)^{T}
\end{array}\right)
$$

В частности, распределение $\mathscr{D}_{\Phi}^{\imath}$ инвариантно относительно этого оператора, причем $\left.\mathscr{B}\right|_{\mathscr{D}_{\Phi}^{\imath}}=B$. Кроме того, $\operatorname{Im} \mathscr{B} \subset \mathscr{L}, \mathscr{M} \subset \operatorname{ker} \mathscr{B}$.

ПРЕДЛОЖЕНИЕ 4. Оператор Я̈В обладает следующими свойствами:

$$
\begin{gathered}
\mathscr{B} \circ \Phi=\Phi \circ \mathscr{B}, \\
\langle\mathscr{B}(X), Y\rangle=-\langle X, \mathscr{B} Y\rangle \quad(X, Y \in \mathfrak{X}(M)) .
\end{gathered}
$$

ДокАЗАТЕльство немедленно следует из (30) и (1). Например,

$$
\langle\mathscr{B} X, Y\rangle=g_{i j} \mathscr{B}_{j}^{i} X^{j} Y_{i}=B_{b}^{a} X^{b} Y_{a}+B_{\widehat{b}}^{\widehat{a}} X^{\widehat{b}} Y_{\widehat{a}}=B_{b}^{a} X^{b} Y_{a}-B_{a}^{b} X_{b} Y^{a}
$$

Аналогично

$$
\langle X, \mathscr{B} Y\rangle=g_{i j} \mathscr{B}_{k}^{j} X^{i} Y^{k}=B_{b}^{a} Y^{b} X_{a}+B_{\widehat{b}}^{\widehat{a}} Y^{\widehat{b}} X_{\widehat{a}}=B_{b}^{a} Y^{b} X_{a}-B_{a}^{b} Y_{b} X^{a}
$$

откуда следует $\left(31_{2}\right)$. Точно так же доказывается и $\left(31_{1}\right)$. 
ПРЕДЛОЖЕНИЕ 5. На пространстве присоединенной G-структурь тензор $\nabla \mathscr{B}$ имеет следующие компоненты:

$$
\begin{aligned}
& \mathscr{B}_{b, c}^{a}=B_{b c}^{a}, \\
& \mathscr{B}_{b, \widehat{c}}^{a}=B_{b}^{a c}, \\
& \mathscr{B}_{0, b}^{a}=-B_{c}^{a} B_{b}^{c}, \quad \mathscr{B}_{b, \widehat{c}}^{0}=B_{b}^{h} B_{h}^{c},
\end{aligned}
$$

плюс соотношения, полученные с учетом вещественности әтого тензора. Остальные компоненты этого тензора нулевые.

ДокАЗАТЕЛЬСТво. Поскольку $\mathscr{B}$ - тензор типа $(1,1)$, его компоненты на пространстве расслоения реперов многообразия $M$ удовлетворяют уравнениям

$$
d \mathscr{B}_{j}^{i}+\mathscr{B}_{j}^{k} \omega_{k}^{i}-\mathscr{B}_{k}^{i} \omega_{j}^{k}=\mathscr{B}_{j, k}^{i} \omega^{k} .
$$

Расписывая эти соотношения на пространстве присоединенной $G$-структуры с учетом (21), (25) и (30), ввиду линейной независимости базисных форм получим (32).

Выясним геометрический смысл соотношений $\left(24_{2}\right),\left(24_{3}\right)$, проведя над ними процедуру восстановления (см. (13)-(15)). Прежде всего отметим, что в силу вещественности тензора $R$ и ввиду (22) эти соотношения равносильны, получаясь друг из друга операцией комплексного сопряжения. С учетом предложения 3 , a также соотношений $(24),(26)$ и последуюших замечаний имеем: $R_{b 0 c}^{a}=\mathscr{B}_{b, c}^{a}$, $R_{b 0 c}^{\widehat{a}}=\mathscr{B}_{b, c}^{\widehat{a}}=0, R_{b 0 c}^{0}=\mathscr{B}_{b, c}^{0}=0$. Таким образом, $R_{b 0 c}^{i}=\mathscr{B}_{b, c}^{i}$, а значит, в каждой точке $p \in M$

$$
R\left(\varepsilon_{0}, \varepsilon_{c}\right) \varepsilon_{b}=\nabla_{\varepsilon_{c}}(\mathscr{B}) \varepsilon_{b} .
$$

Поскольку $\xi_{p}=\varepsilon_{0}$, а векторы $\left\{\varepsilon_{a}\right\}$ образуют базис подпространства $\left(\mathscr{D}_{\Phi}^{\imath}\right)_{p}$, последнее соотношение равносильно тождеству

$$
R(\xi, X) Y=\nabla_{X}(\mathscr{B}) Y, \quad X, Y \in \mathscr{D}_{\Phi}^{2} .
$$

Учитывая, что эндоморфизм $\sigma=\frac{1}{2}(\mathrm{id}-\sqrt{-1} \Phi)$ является проектором модуля $\mathscr{L}^{c}$ на подмодуль $\mathscr{D}_{\Phi}^{\imath}$, тождество (33) можно переписать в форме

$$
R(\xi, X-\sqrt{-1} \Phi(X))(Y-\sqrt{-1} \Phi(Y))=\nabla_{X-\sqrt{-1}} \Phi(X)(\Phi)(Y-\sqrt{-1} \Phi(Y)),
$$

где $X \in \mathscr{L}$. Раскрьвая это соотношениепо линейности и выделяя действительную и мнимую части полученного равенства, приходим к эквивалентному тождеству:

$$
R(\xi, X) Y-R(\xi, \Phi X) \Phi Y=\nabla_{X}(\mathscr{B}) Y-\nabla_{\Phi X}(\mathscr{B}) \Phi Y, \quad X, Y \in \mathscr{L} .
$$

Наконец, поскольку эндоморфизм $\mathbf{l}=-\Phi^{2}$ является проектором на распределение $\mathscr{L}$, получаем, что последнее тождество равносильно тождеству

$$
R\left(\xi, \Phi^{2} X\right) \Phi^{2} Y-R(\xi, \Phi X) \Phi Y=\nabla_{\Phi^{2} X}(\mathscr{B}) \Phi^{2} Y-\nabla_{\Phi X}(\mathscr{B}) \Phi Y,
$$

где $X, Y \in \mathfrak{X}(M)$ - произвольные векторные поля. Доказана 
Теорема 2. Тензор Римана-Кристоффеля $Q \mathscr{S}$-многообразия обладает дополнительным свойством симметрии, вырахсенным тохсдеством (34).

ЗАмЕчАниЕ 2. С учетом (32) непосредственно проверяется, что функции системы $\left\{B_{a b}^{c}, \overline{B_{a b}^{c}}=-B_{c}^{a b}\right\}$ служат компонентами вешественного тензора

$$
\mathbf{B}(X, Y)=\frac{1}{2}\left(\nabla_{\Phi^{2} X}(\Phi)\left(\Phi^{2} Y\right)-\nabla_{\Phi X}(\Phi)(\Phi Y)\right)
$$

Из (34) вытекает тождество

$$
\mathbf{B}(X, Y)=\frac{1}{2}\left(R\left(\xi, \Phi^{2} X\right) \Phi^{2} Y-R(\xi, \Phi X) \Phi Y\right)
$$

которое в известной мере определяет геометрический смысл тензора $\mathbf{B}$.

ОПРЕДЕЛЕНИЕ 2. Скажем, что $Q \mathscr{S}$-многообразие $M$ удовлетворяет первому дополнительному тождеству кривизны, или является многообразием класса $C R_{1}$, если

$$
R\left(\xi, \Phi^{2} X\right) \Phi^{2} Y-R(\xi, \Phi X) \Phi Y=0, \quad X, Y \in \mathfrak{X}(M) .
$$

ТЕОРема 3. QS -многообразие $M$ является многообразием класса $C R_{1}$ тогда и только тогда, когда на нем $\mathbf{B}=0$ или, что равносильно, на $M$ вьполнено тождество

$$
\nabla_{\Phi^{2} X}(\mathscr{B}) \Phi^{2} X-\nabla_{\Phi X}(\mathscr{B}) \Phi X=0, \quad X \in \mathfrak{X}(M) .
$$

ДокАЗАТЕльство. Из (34) следует, что многообразие $M$ принадлежит классу $C R_{1}$ тогда и только тогда, когда на нем выполняется тождество

$$
\nabla_{\Phi^{2} X}(\mathscr{B}) \Phi^{2} Y-\nabla_{\Phi X}(\mathscr{B}) \Phi Y=0, \quad X, Y \in \mathfrak{X}(M)
$$

т.е. $\mathbf{B}=0$. $\mathbf{C}$ другой стороны, проводя процедуру восстановления соотношения $\left(22_{1}\right)$ с учетом $\left(32_{1}\right),\left(32_{2}\right)$ и замечания 2 , убеждаемся, что тензор, стоящий в левой части тождества (37), симметричен, а следовательно, равенство нулю этого тензора равносильно равенству нулю его симметризации, что, в свою очередь, равносильно тождеству (36).

В качестве приложения этого результата приведем

ПРЕДЛОЖЕНИЕ 6. Сасакиево и косимплектическое многообразия являются многообразиями класса $C R_{1}$. 
ДокАЗАТЕльство. Заметим, что в силу равенств (1) и предложения 2 для сасакиевых и косимплектических структур $\mathscr{B}=\varepsilon \Phi$, где $\varepsilon=-1$ либо 0 соответственно. Но в силу нормальности обоих видов структур тождества (37), а тем более (36), вьполнены в силу предложения 1.

Другой важньй пример $Q \mathscr{S}$-многообразий класса $C R_{1}$ дают пространства канонических главных $T^{1}$-расслоений над келеровыми многообразиями. Характеристический класс таких главных расслоений порожден формой Риччи [19]. Прямьм подсчетом можно убедиться, что почти контактная метрическая структура, канонически индуцированная на пространстве такого расслоения, действительно является $Q \mathscr{S}$-структурой, причем выполнено тождество (36).

Пусть $M-Q \mathscr{S}$-многообразие класса $C R_{1}$. Заметим, что тождество $(37)$ на пространстве присоединенной $G$-структуры равносильно тождествам

$$
B_{b c}^{a}=0, \quad B_{b}^{a c}=0 .
$$

С учетом этого, проводя процедуру восстановления соотношений (32), убеждаемся, что они равносильны тождеству

$$
\nabla_{X}(\mathscr{B}) Y=\left\langle\mathscr{B}^{2} X, Y\right\rangle \xi-\eta(Y) \mathscr{B}^{2} X, \quad X, Y \in \mathfrak{X}(M) .
$$

Пусть $M-Q \mathscr{S}$-многообразие. Введем в рассмотрение две связности на $M$ : связность

$$
\widehat{\nabla}_{X} Y=\nabla_{X} Y-\eta(X) \mathscr{B}(Y)
$$

(первая каноническая связность) и связность

$$
\widetilde{\nabla}_{X} Y=\nabla_{X} Y+\langle\mathscr{B}(X), Y\rangle \xi-\mathscr{B}(X) \eta(Y)
$$

(вторая каноническая связность). Первая каноническая связность замечательна тем, что в ней первая группа структурных уравнений (18) записывается в форме

$$
d \omega=2 B_{b}^{a} \omega^{b} \wedge \omega_{a}, \quad d \omega^{a}=\zeta_{b}^{a} \wedge \omega^{b}, \quad d \omega_{a}=-\zeta_{a}^{b} \wedge \omega_{b},
$$

где $\left\{\zeta_{j}^{i}\right\}$ - компоненты формы первой канонической связности. При этом $\zeta_{b}^{a}=\omega_{b}^{a}+$ $B_{b}^{a} \omega, \zeta_{\widehat{b}}^{\widehat{a}}=-\zeta_{a}^{b}, \zeta_{b}^{\widehat{a}}=\omega_{b}^{\widehat{a}}, \zeta_{\widehat{b}}^{a}=\omega_{\widehat{b}}^{a}$. Основное свойство второй канонической связности определяется следующим утверждением.

ПРЕДЛОЖЕНИЕ 7. Тензоры $\mathscr{B}, \Psi, \xi, \eta, g$ Q⿻ параллельны во второй канонической связности.

ДокАЗАТЕльство. Прежде всего заметим, что из (30) непосредственно следует, что $\eta \circ \mathscr{B}=0$ и $\mathscr{B}(\xi)=0$. Далее, если обозначить

$$
T(X, Y)=\langle\mathscr{B}(X), Y\rangle \xi-\mathscr{B}(X) \eta(Y),
$$

то $T$ - это тензор аффинной деформации от римановой связности к первой канонической связности. Следовательно,

$$
\widetilde{\nabla}_{X}(\mathscr{B}) Y=\nabla_{X}(\mathscr{B}) Y+T(X, \mathscr{B}(Y))-\mathscr{B} \circ T(X, Y) .
$$

Подставляя сюда (39) и (40), с учетом с деланных замечаний и ввиду $\left(31_{2}\right)$ получаем, что $\widetilde{\nabla}_{X}(\mathscr{B}) Y=0, X, Y \in \mathfrak{X}(M)$. Аналогично доказьвается параллельность остальных тензоров. 
ПрЕДЛОЖЕНИЕ 8. Во введенных обозначениях $\mathscr{L}=\mathscr{D}^{\prime} \oplus \mathscr{D}^{\prime \prime}$, причем подмодули $\mathscr{D}^{\prime}=\operatorname{Im} \mathscr{B}$ и $\mathscr{D}^{\prime \prime}=\left.\operatorname{ker} \mathscr{B}\right|_{\mathscr{L}}$ взаимно ортогональны и являются распределениями определенной размерности на многообразии $M$, инвариантными относительно действия эндоморфизмов $\Phi$ и $\mathscr{B}$.

Доказательство немедленно вытекает из (31) и из предложения 7.

В частности, корректно следующее

ОПРЕДЕЛЕНИЕ 3. Рангом $Q \mathscr{S}$-структуры на многообразии $M$ назовем число $r=\operatorname{rank} \mathscr{B}=\operatorname{dim} \mathscr{D}^{\prime}$. Если $\operatorname{rank} \mathscr{B}=\operatorname{dim} M-1$, многообразие $M$ назовем Q⿻्S-многообразием максимального ранга.

Например, из предложения 2 вытекает, что многообразие Сасаки является $Q \mathscr{S}$-многообразием максимального ранга.

ЗАмечАниЕ 3 . Аналогично предложению 8 доказывается, что для $Q \mathscr{S}$-многообразия класса $C R_{1}$

$$
\mathscr{L}=\bigoplus_{k=1}^{m} \mathscr{D}_{\lambda_{k}},
$$

где $\mathscr{D}_{\lambda_{k}}=\left(\mathscr{D}_{\mathscr{B}}^{\lambda_{k}} \oplus \mathscr{D}_{\mathscr{B}}^{-\lambda_{k}}\right) \cap \mathscr{L}, \lambda_{1}, \ldots, \lambda_{m}$ - попарно различные по модулю собственные значения эндоморфизма $\mathscr{B}, \mathscr{D}_{\mathscr{B}}^{\lambda_{k}}$ - собственный подмодуль эндоморфизма $\mathscr{B}$, отвечающий собственному значению $\lambda_{k}$. При этом $\mathscr{D}_{\lambda_{i}} \perp \mathscr{D}_{\lambda_{j}}(i \neq j)$.

ТЕОРема 4. $Q \mathscr{S}$-многообразие $M$ класса $C R_{1}$ либо является косимплектическим многообразием, либо локально әквивалентно произведению $Q \mathscr{S}$-многообразия максимального ранга и келерова многообразия.

ДокАЗАТЕльство. Из предложения 8 вытекает, что присоединенная $G$-структура многообразия $M$ допускает редукцию по подгруппе $\{1\} \times U(r) \times U(s)$, где $2 r=\operatorname{dim} \mathscr{D}^{\prime}, 2 s=\operatorname{dim} \mathscr{D}^{\prime \prime}$. Пространство сокращенной $G$-структуры состоит из $A$-реперов, векторы $\varepsilon_{1}, \ldots, \varepsilon_{r}$ которых принадлежат комплексификации подпространства $\left(\mathscr{D}^{\prime}\right)_{p}$, а векторы $\varepsilon_{r+1}, \ldots, \varepsilon_{n}$ - комплексификации подпространства $\left(\mathscr{D}^{\prime \prime}\right)_{p}$, где $p \in M$ - вершина репера. Условимся, что индексы $\alpha, \beta, \gamma, \ldots$ пробегают значения от 1 до $r$, индексы $\rho, \sigma, \tau-$ значения от 0 до $r$, а индексы $\varphi, \psi, \chi, \ldots$ - значения от $r+1$ до $n$. Очевидно, на пространстве сокрашенной $G$-структуры $B_{\varphi}^{\alpha}=0, B_{\alpha}^{\varphi}=0, B_{\psi}^{\varphi}=0$. Дифференцируя эти соотношения внешним образом с учетом $(21),(38)$ и невырожденности оператора $\left.\mathscr{B}\right|_{\mathscr{D}^{\prime}}$, получаем, что $\omega_{\varphi}^{\alpha}=0$, $\omega_{\alpha}^{\varphi}=0$. С учетом этих замечаний структурные уравнения $(18),(19)$ на пространстве сокращенной $G$-структуры распадутся на две групшы:

$$
\begin{aligned}
d \omega= & 2 B_{\beta}^{\alpha} \omega^{\beta} \wedge \omega_{\alpha}, \\
d \omega^{\alpha}= & \omega_{\beta}^{\alpha} \wedge \omega^{\beta}+B_{\beta}^{\alpha} \omega \wedge \omega^{\beta}, \\
d \omega_{\alpha}= & -\omega_{\alpha}^{\beta} \wedge \omega_{\beta}-B_{\alpha}^{\beta} \omega \wedge \omega_{\beta}, \\
d \omega_{\beta}^{\alpha}= & \omega_{\gamma}^{\alpha} \wedge \omega_{\beta}^{\gamma}+\left(A_{\beta \gamma}^{\alpha \delta}-2 B_{\beta}^{\alpha} B_{\gamma}^{\delta}\right) \omega^{\gamma} \wedge \omega_{\delta} \\
& +B_{\beta \gamma}^{\alpha} \omega \wedge \omega^{\gamma}+B_{d}^{\alpha \gamma} \omega \wedge \omega_{\gamma},
\end{aligned}
$$




$$
\begin{aligned}
& d \omega^{\varphi}=\omega_{\psi}^{\varphi} \wedge \omega^{\psi}, \\
& d \omega_{\varphi}=-\omega_{\varphi}^{\psi} \wedge \omega_{\psi}, \\
& d \omega_{\psi}^{\varphi}=\omega_{\chi}^{\varphi} \wedge \omega_{\psi}^{\chi}+A_{\psi \chi}^{\varphi \tau} \omega^{\chi} \wedge \omega_{\tau} .
\end{aligned}
$$

Отсюда следует, что распределения $\mathscr{D}^{\prime}$ и $\mathscr{D}^{\prime \prime}$ вполне интегрируемы. Зафиксируем какое-нибудь локальное сечение $s$ тотального пространства присоединенной $G$-структуры и отождествим формы $\omega_{j}^{i}$ и $\omega^{i}$ с их антиувлечениями $s^{*} \omega_{j}^{i}$ и $s^{*} \omega^{i}$ на $M$ соответственно. Тогда формы $\left\{\omega^{\varphi}\right\}$ и $\left\{\omega^{\alpha}, \omega\right\}$, а также им комплексно сопряженные, очевидно, локально образуют системы Пфаффа (комплексификации) распределений $\mathscr{D}^{\prime}$ и $\mathscr{D}^{\prime \prime}$ соответственно. Соотношения $(41),(42)$ показывают, что эти распределения вполне интегрируемы. В силу предложения 8 на интегральных многообразиях максимальной размерности этих систем индуцируются почти контактная метрическая и почти эрмитова структуры соответственно (а именно ограничения исходной почти контактной метрической структуры), причем опять-таки в силу соотношений $(41),(42)$ эти структуры являются $Q \mathscr{S}$-структурой и келеровой структурой соответственно. Пусть $(U, \varphi)$ - локальная карта многообразия $M$ с координатами $\left\{x^{0}, \ldots, x^{2 n}\right\}$. Тогда отображение $\varphi-$ диффеоморфизм области $U \subset M$ на область $V=\varphi(U) \subset \mathbb{R}^{2 n+1}$ и, следовательно, $\varphi$ определяет отображение увлечения $\varphi_{*}$ тензорной алгебры многообразия $U$. Применяя это отображение к системам форм $\left\{\omega^{\varphi}\right\}$ и $\left\{\omega^{\alpha}, \omega\right\}$ на $U$, получаем системы форм $\left\{\vartheta^{\varphi}\right\}$ и $\left\{\vartheta^{\rho}\right\}$ на $V$, удовлетворяющих уравнениям вида $d \vartheta^{\varphi}=\vartheta_{\psi}^{\varphi} \wedge \vartheta^{\psi}$ и $d \vartheta^{\rho}=\vartheta_{\sigma}^{\rho} \wedge \vartheta^{\sigma}$ соответственно. Из классической теоремы Фробениуса следует, что существует система $u^{i}=u^{i}\left(x^{0}, \ldots, x^{2 n}\right)$ гладких функций на $V$ таких, что

$$
\vartheta^{\rho}=A_{\sigma}^{\rho} d u^{\sigma}, \quad \vartheta^{\varphi}=A_{\psi}^{\varphi} d u^{\psi}
$$

где $\left(A_{\sigma}^{\rho}\right)$ и $\left(A_{\psi}^{\varphi}\right)$ - матрицы гладких функций, невырожденные в каждой точке области определения. Отсюда, в частности, вытекает, что матрица

$$
\left(A_{j}^{i}\right)=\left(\begin{array}{cc}
A_{\sigma}^{\rho} & 0 \\
0 & A_{\psi}^{\varphi}
\end{array}\right)
$$

также невырождена в каждой точке области определения, а система функций $\left\{u^{i}=u^{i} \circ \varphi(p): p \in U\right\}$ определяет допустимые карты $(U, \phi)$ на $M$, которые назовем специальными. При этом системы функций $\left\{u^{\rho}\right\}$ и $\left\{u^{\varphi}\right\}$ определяют допустимые карты $\left(U^{\prime},\left.\phi\right|_{U^{\prime}}\right)$ и $\left(U^{\prime \prime},\left.\phi\right|_{U^{\prime \prime}}\right)$ на интегральных многообразиях распределений $\mathscr{D}^{\prime}$ и $\mathscr{D}^{\prime \prime}$ соответственно. Фиксируем точку $m_{0} \in U$. Пусть $\phi(u)=\left(u_{0}^{0}, \ldots, u_{0}^{2 n}\right)$. Без ограничения общности можно считать, что область $V=\phi(U)$ является кубической, т.е. задается системой неравенств

$$
\left|u^{i}-u_{0}^{i}\right|<\alpha, \quad i=0, \ldots, 2 n, \quad \alpha \in \mathbb{R}^{2 n+1}
$$

Пусть $W_{0}^{\prime}=U^{\prime}$ и $W_{0}^{\prime \prime}=U^{\prime \prime}$ - интегральные многообразия распределений $\mathscr{D}^{\prime}$ и $\mathscr{D}^{\prime \prime}$ соответственно, проходящие через точку $m_{0}$. Они задаются уравнениями $u^{\varphi}=u_{0}^{\varphi}$ $(\varphi=2 r+1, \ldots, 2 n)$ и $u^{\rho}=u_{0}^{\rho}(\rho=0, \ldots, 2 r)$ соответственно. Пусть $m \in U-$ произвольная точка, $\phi(m)=\left(u^{1}, \ldots, u^{2 n+1}\right), W^{\prime}$ и $W^{\prime \prime}$ - интегральные многообразия этих распределений, проходящие через точку $m$. Построим точки $m^{\prime}=W_{0}^{\prime} \cap W^{\prime \prime} \in$ 
$W_{0}^{\prime}$ и $m^{\prime \prime}=W_{0}^{\prime \prime} \cap W^{\prime} \in W_{0}^{\prime \prime}$ с координатами $m^{\prime}\left(u^{0}, \ldots, u^{2 r}, u_{0}^{2 r+1}, \ldots, u_{0}^{2 n}\right)$ и $m^{\prime \prime}\left(u_{0}^{0}, \ldots, u_{0}^{2 r}, u^{2 r+1}, \ldots, u^{2 n}\right)$ соответственно. Тем самым построено отображение $f_{U}: U \rightarrow U^{\prime} \times U^{\prime \prime}$, которое, очевидно, является диффеоморфизмом. Оно индуцирует в каждой точке $m \in U$ изоморфизмы $\left.\left(f_{*}\right)_{m}\right|_{T_{m}\left(W^{\prime}\right)}: T_{m}\left(W^{\prime}\right) \rightarrow T_{m^{\prime}}\left(U^{\prime}\right)$ и $\left.\left(f_{*}\right)_{m}\right|_{T_{m}\left(W^{\prime \prime}\right)}: T_{m}\left(W^{\prime \prime}\right) \rightarrow T_{m^{\prime \prime}}\left(U^{\prime \prime}\right)$. Эти изоморфизмы индуцируют на многообразиях $U^{\prime}$ и $U^{\prime \prime}$ почти контактную метрическую структуру $\left(\Phi^{\prime}, \xi^{\prime}, \eta^{\prime}, g^{\prime}\right)$ и почти эрмитову структуру $\left(J^{\prime \prime}, g^{\prime \prime}\right)$ соответственно, которые являются увлечениями соответствующих структур $\left(\left.\Phi\right|_{W^{\prime}},\left.\xi\right|_{W^{\prime}},\left.\eta\right|_{W^{\prime}},\left.g\right|_{W^{\prime}}\right)$ и $\left(\left.\Phi\right|_{W^{\prime \prime}},\left.g\right|_{W^{\prime \prime}}\right)$. Покажем, что в действительности эти структуры не зависят от выбора точки $m_{0}$, а значит, внутренним образом определяют соответствующие структуры на многообразиях $U^{\prime}$ и $U^{\prime \prime}$. Из построения диффеоморфизма $f_{U}$ видно, что для этого достаточно доказать, что компоненты $\Phi_{\sigma}^{\rho}, \xi^{\rho}, \eta_{\rho}, g_{\rho \sigma}$ и $\Phi_{\psi}^{\varphi}, g_{\varphi \psi}$ ограниченных на интегральных многообразиях $W^{\prime}$ и $W^{\prime \prime}$ структур не зависят от переменных $u^{\varphi}$ и $u^{\rho}$ соответственно. Заметим, что в силу соотношений $\omega_{\varphi}^{\rho}=\omega_{\rho}^{\varphi}=0$ компоненты обобщенных символов Кристоффеля $\Gamma_{j k}^{i}$ второй канонической связности в специальной карте $(U, \phi)$ будут равны нулю, если среди их индексов $i, j$ есть как индексы, пробегающие значения от 0 до $2 r$, так и индексы, пробегающие значения от $2 r+1$ до $2 n$. Далее, в силу параллельности тензора $\Phi$ во второй канонической связности $\widetilde{\nabla} \Phi=0$, в частности

$$
0=\widetilde{\nabla}_{\varphi} \Phi_{\sigma}^{\rho}=\frac{\partial \Phi_{\sigma}^{\rho}}{\partial u^{\varphi}}-\Phi_{\tau}^{\rho} \Gamma_{\sigma \varphi}^{\tau}+\Phi_{\beta}^{\tau} \Gamma_{\tau \varphi}^{\rho}=\frac{\partial \Phi_{\sigma}^{\rho}}{\partial u^{\varphi}}
$$

откуда вытекает, что $\frac{\partial \Phi_{\sigma}^{\rho}}{\partial u^{\varphi}}=0$. Аналогично $\frac{\partial \Phi_{\psi}^{\varphi}}{\partial u^{\rho}}=0$, что и требовалось доказать. Точно такое же рассуждение с учетом предложения 7 проводится и для метрического тензора, а также вектора $\xi$ и ковектора $\eta$. Следовательно, на многообразиях $U^{\prime}$ и $U^{\prime \prime}$ отображением $f_{U}$ корректно индуцируются почти контактная метрическая структура и почти эрмитова структура соответственно, относительно которых отображение $f_{U}$ является эквивалентностью. Как мы уже видели, индуцированные на $W^{\prime}$ и $W^{\prime \prime}$ структуры являются $Q \mathscr{S}$-структурой (максимального ранга) и келеровой структурой соответственно. Следовательно, такими же будут и структуры, индуцированные на $U^{\prime}$ и $U^{\prime \prime}$ соответственно. Заметим также, что, как следует из классической теоремы де-Рама [17], если многообразие $M$ полно и односвязно, построенные выше локальные изометрии могут быть определены глобально.

Пусть $M-Q \mathscr{S}$-многообразие максимального ранга. В этом случае $\mathscr{D}^{\prime}=\mathscr{L}$, а значит, $\mathscr{D}^{\prime \prime}=\{0\}, \operatorname{ker} \mathscr{B}=\mathscr{M}$. Определим на многообразии $M$ билинейную форму

$$
G(X, Y)=-\langle\mathscr{B} X, \Phi Y\rangle+\eta(X) \eta(Y), \quad X, Y \in \mathfrak{X}(M) .
$$

ПРЕДЛОЖЕНИЕ 9. На $Q \mathscr{S}$-многообразии $M$ максимального ранга форма $G$ задает псевдориманову структуру.

ДокАЗАтЕльство. Из предложения 4 легко следует, что форма $G$ симметрична. Докажем ее невырожденность. Пусть $G(X, Y)=0$ для любого $Y \in \mathfrak{X}(M)$. В частности, $G(X, \widetilde{Y})=0(\widetilde{Y} \in \mathscr{L})$. Но тогда $\langle\mathscr{B} \circ \Phi(X), Y\rangle=0$ и в силу невырожденности метрики $\langle\cdot, \cdot\rangle$ на $\mathscr{L}$ заключаем, что $\mathscr{B} \circ \Phi(X)=0 . \operatorname{Ho} \operatorname{ker}(\mathscr{B} \circ \Phi)=\mathscr{M}$, откуда вытекает, что $X=\alpha \xi, \alpha \in \mathbb{R}$. Следовательно, $0=G(X, Y)=\alpha \eta(\xi) \eta(Y)=$ $\alpha \eta(Y)$, а поскольку $\eta \neq 0$, имеем $\alpha=0$, т.е. $X=0$. 
ЗАмЕчАниЕ 4. Из предложения 2 вытекает, что в случае многообразия Сасаки метрика $G$ совпадает с исходной метрикой $g$ многообразия.

ПРЕДЛОЖЕНИЕ 10. Во введенных обозначениях $\mathscr{S}=(\Phi, \xi, \eta, G)$ - сасакиева структура на $M$.

ДокАЗАТЕЛЬСтво. Очевидно, первые четыре аксиомы почти контактной метрической структуры для $\mathscr{S}$ выполнены. Далее,

$$
\begin{aligned}
G(\Phi X, \Phi Y) & =-\left\langle\Phi \circ \mathscr{B}(X), \Phi^{2}(Y)\right\rangle+\eta(\Phi X) \eta(\Phi Y) \\
& =\left\langle\mathscr{B} X, \Phi^{3} Y\right\rangle+0=-\langle\mathscr{B} X, \Phi Y\rangle=G(X, Y)-\eta(X) \eta(Y)
\end{aligned}
$$

Таким образом, $\mathscr{S}$-почти контактная метрическая структура. Очевидно, она нормальна, поскольку отличается от исходной структуры только метрикой. Наконец, $d \eta=\widetilde{\Omega}$, где $\widetilde{\Omega}-$ фундаментальная форма почти контактной метрической структуры $\mathscr{S}$. В самом деле, из (18) следует, что $d \eta=d \omega=2 B_{b}^{a} \omega^{b} \wedge \omega_{a}$. С другой стороны, в силу предложения 4

$$
\begin{aligned}
\widetilde{\Omega}(X, Y) & =-\left\langle\mathscr{B} X, \Phi^{2} Y\right\rangle+\eta(X) \eta(\Phi Y)=-\left\langle\mathscr{B} X, \Phi^{2} Y\right\rangle \\
& =\langle\Phi \circ \mathscr{B}(X), \Phi Y\rangle=\langle\mathscr{B} X, Y\rangle-\eta(\mathscr{B} X) \eta(Y)=\langle\mathscr{B} X, Y\rangle .
\end{aligned}
$$

Значит, в произвольном репере $\widetilde{\Omega}_{i j}=-g_{k j} \mathscr{B}_{i}^{k}$. В частности, в $A$-репере

$$
\widetilde{\Omega}_{a b}=\widetilde{\Omega}_{\widehat{a} \widehat{b}}=\widetilde{\Omega}_{a 0}=\widetilde{\Omega}_{\widehat{a} 0}=0, \quad \widetilde{\Omega}_{a \widehat{b}}=g_{c \widehat{b}} \mathscr{B}_{a}^{c}=\mathscr{B}_{a}^{b}
$$

Следовательно, $\widetilde{\Omega}=\Omega_{i j} \omega^{i} \otimes \omega^{j}=2 \Omega_{a \widehat{b}} \omega^{a} \wedge \omega^{\widehat{b}}=2 B_{a}^{b} \omega^{a} \wedge \omega_{b}=d \eta$. Таким образом, $\mathscr{S}$ - нормальная контактная метрическая, т.е. сасакиева структура.

ОПРЕДЕЛЕНИЕ 4 . Переход от метрики $g=\langle\cdot, \cdot\rangle$ к метрике $G$, заданной соотношением (43), назовем $\mathscr{B}$-преобразованием $Q \mathscr{S}$-структуры максимального ранга. В случае, когда $M-Q \mathscr{S}$-структура класса $C R_{1}$, ее $\mathscr{B}$-преобразованием назовем переход от метрики $g=\langle\cdot, \cdot\rangle$ к метрике $\mathscr{G}=\left.G\right|_{\mathscr{D}^{\prime} \oplus \mathscr{M}}+\left.g\right|_{\mathscr{D}^{\prime \prime}}$.

ПримеР 1. Пусть $\mathscr{S}=(\Phi, \xi, \eta, g)$ - почти контактная метрическая структура. Перейдем от нее к новой структуре $\widetilde{\mathscr{S}}=\left(\Phi, \frac{1}{\alpha} \xi, \alpha \eta, \alpha^{2} g\right)$, где $\alpha$ - ненулевая константа. Очевидно, $\widetilde{\mathscr{S}}$ - почти контактная метрическая структура. Указанное преобразование почти контактной метрической структуры называется гомотетией $c$ коэффициентом $\alpha$. Очевидно, почти контактная метрическая структура, гомотетичная сасакиевой, является $Q \mathscr{S}$-структурой максимального ранга. Более того, с учетом определения оператора $\mathscr{B}$ и в силу предложения 2 она однозначно характеризуется соотношением $\mathscr{B}=-\frac{1}{\alpha} \Phi$, или

$$
B_{b}^{a}=-\frac{\sqrt{-1}}{\alpha} \delta_{b}^{a} .
$$

$\mathscr{B}$-преобразование такой структуры приводит к новой сасакиевой структуре $\widehat{\mathscr{S}}=$ $\left(\Phi, \frac{1}{\alpha} \xi, \alpha \eta, G\right)$ с метрикой

$$
\begin{aligned}
G(X, Y) & =-\alpha^{2} g(\mathscr{B} X, \Phi Y)+\alpha^{2} \eta(X) \eta(Y)=\alpha^{2} \frac{1}{\alpha} g(\Phi X, \Phi Y)+\alpha^{2} \eta(X) \eta(Y) \\
& =\alpha(g(X, Y)-\eta(X) \eta(Y))+\alpha^{2} \eta(X) \eta(Y) \\
& =\alpha g(X, Y)+\alpha(\alpha-1) \eta(X) \eta(Y)
\end{aligned}
$$


Переход от структуры $\mathscr{S}$ к структуре $\widehat{\mathscr{S}}$ известен как обобщенное преобразование $D$-гомотетии (преобразование $D$-гомотетии при $\alpha>0$ и преобразование $D$-инверсии при $\alpha<0)$ [6] (см. также [14]). Таким образом, доказана

ТеОРема 5. Обобщенное преобразование D-гомотетии сасакиевой структуры является композицией гомотетии и $\mathscr{B}$-преобразования.

С учетом приведенных определений, теоремы 4 и предложения 10 получаем следующую теорему.

TEOPEма 6. QS -многообразие класса $C R_{1}$ либо является косимплектическим многообразием, либо с точностью до $\mathscr{B}$-преобразования структурь локально әквивалентно произведению сасакиева и келерова многообразий.

Пусть $M-Q \mathscr{S}$-многообразие максимального ранга. Используя характеристические свойства римановой связности (отсутствие кручения и параллельность метрического тензора), нетрудно вычислить тензор

$$
h(X, Y)=\nabla_{X}^{*} Y-\nabla_{X} Y
$$

аффинной деформации от римановой связности $\nabla$ метрики $g$ к римановой связности $\nabla^{*}$ метрики $G$ :

$$
h=-\eta \otimes(\mathscr{B}+\Phi)-(\mathscr{B}+\Phi) \otimes \eta .
$$

С учетом (1) и (30) очевидно, что соотношение (44) в $A$-репере (т.е. на пространстве присоединенной $G$-структуры) выражается тождествами

$$
h_{0 a}^{b}=h_{a 0}^{b}=-\left(B_{a}^{b}+\sqrt{-1} \delta_{a}^{b}\right),
$$

плюс соотношения, полученные с учетом вешественности тензора $h$. Остальные компоненты этого тензора нулевые.

ЛЕмма 1. Пусть $\left\{\omega_{j}^{i}\right\}$ u $\left\{\theta_{j}^{i}\right\}-$ компоненты форм связностей $\nabla u \nabla^{*}$ на многообразии $M$ соответственно, $\left\{h_{j k}^{i}\right\}$ - компоненты тензора $h(X, Y)=$ $\nabla_{X}^{*} Y-\nabla_{X} Y$ аффинной деформации от связности $\nabla$ квязности $\nabla^{*}$. Тогда

$$
\theta_{j}^{i}=\omega_{j}^{i}-h_{k j}^{i} \omega^{k} .
$$

ДокаЗАТЕЛЬСтво. Пусть $X, Y \in \mathfrak{X}(M), Y^{i}{ }_{, k}$ и $Y^{i}{ }_{\mid k}-$ компоненты тензоров $\nabla Y$ и $\nabla^{*} Y$ соответственно. Тогда

$$
\begin{aligned}
d Y^{i}-Y^{j} \omega_{j}^{i} & =Y_{, k}^{i} \omega^{k}, \\
d Y^{i}-Y^{j} \theta_{j}^{i} & =Y^{i}{ }_{\mid k} \omega^{k} .
\end{aligned}
$$

С другой стороны, тождество $\nabla_{X}^{*} Y=\nabla_{X} Y+h(X, Y)$ равносильно соотношению $Y^{i}{ }_{\mid k} X^{k}=Y^{i}{ }_{, k} X^{k}+h_{j k}^{i} X^{j} Y^{k}$ и в силу произвола в выборе $X Y^{i}{ }_{\mid k}=Y^{i}{ }_{, k}+h_{k j}^{i} Y^{j}$. Теперь из в $\left(47_{1}\right)$ получаем, что $d Y^{i}-Y^{j}\left(\omega_{j}^{i}-h_{k j}^{i}\right) \omega^{k}=Y^{i}{ }_{\mid k} \omega^{k}$. Сравнивая это равенство с $\left(47_{2}\right)$, в силу линейной независимости базисных форм получаем (46). 
С учетом (45) и (46) имеем, в частности:

$$
\begin{gathered}
\omega_{0}^{a}=\theta_{0}^{a}-\left(B_{b}^{a}+\sqrt{-1} \delta_{b}^{a}\right) \omega^{b}, \quad \omega_{a}^{0}=\theta_{a}^{0}-\left(B_{a}^{b}-\sqrt{-1} \delta_{a}^{b}\right) \omega_{b} \\
\omega_{b}^{a}=\theta_{b}^{a}-\left(B_{b}^{a}+\sqrt{-1} \delta_{b}^{a}\right) \omega .
\end{gathered}
$$

Подставляя эти соотношения в (18) и (19), получаем структурные уравнения структуры $\mathscr{S}$ в связности $\nabla^{*}$ на пространстве присоединенной $G$-структуры:

$$
\begin{aligned}
d \omega & =2 B_{b}^{a} \omega^{b} \wedge \omega_{a}, \\
d \omega^{a} & =\theta_{b}^{a} \wedge \omega^{b}-\sqrt{-1} \omega \wedge \omega^{a} \\
d \omega_{a} & =-\theta_{a}^{b} \wedge \omega_{b}+\sqrt{-1} \omega \wedge \omega_{a} \\
d \theta_{b}^{a} & =\theta_{c}^{a} \wedge \theta_{b}^{c}+\left(A_{b c}^{a d}+2 \sqrt{-1} \delta_{b}^{a} B_{c}^{d}\right) \omega^{c} \wedge \omega_{d} .
\end{aligned}
$$

Теперь перейдем от $G$-структуры, присоединенной к исходной $Q \mathscr{S}$-структуре, пространство которой состоит из $A$-реперов, к новой $G$-структуре, пространство которой состоит из так называемых косых $A$-реперов относительно построенной сасакиевой структуры. Именно рассмотрим автоморфизм $C=(B \circ \Phi)^{-1}$ модуля $\mathscr{D}_{\Phi}^{\imath}$. С его помощью каждому $A$-реперу $r=\left(p, \varepsilon_{a}, \varepsilon^{b}, \varepsilon\right)$ сопоставим репер $\widetilde{r}=\left(p, \zeta_{a}, \zeta^{b}, \varepsilon\right)$, где $\zeta_{a}=\varepsilon_{a}, \zeta^{b}=\tau C \tau\left(\varepsilon^{b}\right), a, b=1, \ldots, n$. Репер вида $\widetilde{r}$ мы и назовем косым $A$-репером. Оператор $C$, очевидно, эрмитов, поэтому $\zeta^{b}=\tau C \varepsilon_{b}=$ $\tau\left(C_{b}^{h} \varepsilon_{h}\right)=\bar{C}_{b}^{h} \tau\left(\varepsilon_{h}\right)=C_{h}^{b} \varepsilon^{h}=-\sqrt{-1}\left(B^{-1}\right)_{h}^{b} \varepsilon^{h}$. Следовательно,

$$
\zeta^{b}=C_{h}^{b} \varepsilon^{h}=-\sqrt{-1}\left(B^{-1}\right)_{h}^{b} \varepsilon^{h} .
$$

С учетом этого для дуального корепера $\left(p, \theta^{a}, \theta_{b}, \omega\right)$ имеем:

$$
\theta^{a}=\omega^{a}, \quad \theta_{b}=\sqrt{-1} B_{b}^{h} \omega_{h} .
$$

С учетом (50) легко проверить, что совокупность всех косых $A$-реперов определяет $G$-структуру на $M$ со структурной группой $\{1\} \times U(n)$, представленной матрицами вида $\left(\begin{array}{ccc}1 & 0 & 0 \\ 0 & A & 0 \\ 0 & 0 & \frac{B A B^{-1}}{B}\end{array}\right)$, где $A \in U(n)$. Эту G-структуру назовем специальной. Очевидно, в косом $A$-репере

$$
\begin{aligned}
G\left(\zeta_{a}, \zeta^{b}\right) & =-\left\langle\mathscr{B} \varepsilon_{a}, \Phi \zeta^{b}\right\rangle=-\left\langle\mathscr{B} \varepsilon_{a}, \tau \circ \Phi \circ \Phi^{-1} \circ B^{-1} \varepsilon_{b}\right\rangle \\
& =-\left\langle\mathscr{B} \varepsilon_{a}, \mathscr{B}^{-1} \varepsilon^{b}\right\rangle=\left\langle\varepsilon_{a}, \mathscr{B} \circ \mathscr{B}^{-1}\left(\varepsilon^{b}\right)\right\rangle=\left\langle\varepsilon_{a}, \varepsilon^{b}\right\rangle=\delta_{b}^{a} .
\end{aligned}
$$

Кроме того, поскольку $\mathscr{D}_{\Phi}^{\imath} \subset \mathfrak{X}(M)$ - вполне изотропный подмодуль, инвариантньй относительно эндоморфизмов $\mathscr{B}$ и $\Phi, G\left(\zeta^{a}, \zeta^{b}\right)=-\left\langle\mathscr{B} \zeta^{a}, \Phi \zeta^{b}\right\rangle=0$. Аналогично $G\left(\zeta_{a}, \zeta_{b}\right)=0$. Наконец,

$$
\begin{aligned}
\Phi\left(\zeta^{a}\right) & =\Phi \circ \tau \circ C \circ \tau\left(\varepsilon^{a}\right)=\Phi \circ \tau \circ C\left(\varepsilon_{a}\right)=\Phi \circ \tau\left(C_{a}^{h} \varepsilon_{h}\right) \\
& =\Phi\left(\bar{C}_{a}^{h} \tau \varepsilon_{h}\right)=\Phi\left(C_{h}^{a} \varepsilon^{h}\right)=C_{h}^{a}\left(-\sqrt{-1} \varepsilon^{h}\right)=-\sqrt{-1} \zeta^{a}
\end{aligned}
$$

Таким образом, в косом $A$-репере выполняются соотношения (1), хотя, вообще говоря, не выполняются условия $\tau\left(\varepsilon_{a}\right)=\varepsilon^{a}$, чем и объясняется название "косой $A$-репер". Важно отметить, что понятие косого $A$-репера совпадает с понятием $A$-репера тогда и только тогда, когда $C=\mathrm{id}$, т.е. $\mathscr{B}=-\Phi$, т.е. $M$ - многообразие Сасаки. Доказано 
ПРЕДЛОЖЕНИЕ 11. QS्S-многообразие максимального ранга является многообразием Сасаки тогда и только тогда, когда присоединенная и специальная G-структурь на этом многообразии совпадают.

Пусть теперь $M-Q \mathscr{S}$-многообразие класса $C R_{1}$. С учетом (18), (21), (38), (49) и (51) подсчитаем первую группу его структурных уравнений на пространстве специальной $G$-структуры:

$$
\begin{aligned}
d \omega & =-2 \sqrt{-1} \theta^{a} \wedge \theta_{a} \\
d \theta^{a} & =\theta_{b}^{a} \wedge \theta^{b}-\sqrt{-1} \omega \wedge \theta^{a} \\
d \theta_{a} & =-\theta_{a}^{b} \wedge \theta_{b}+\sqrt{-1} \omega \wedge \theta_{a} .
\end{aligned}
$$

Как мы уже знаем, дифференциальное продолжение этих уравнений приводит ко второй группе структурных уравнений, имеющей вид:

$$
d \theta_{b}^{a}=\theta_{c}^{a} \wedge \theta_{b}^{c}+\left(\widetilde{A}_{b c}^{a d}+2 \delta_{b}^{a} \delta_{c}^{d}\right) \theta^{c} \wedge \theta_{d}
$$

где $\left\{\widetilde{A}_{b c}^{a d}\right\}$ - семейство функций на пространстве специальной $G$-структуры, служащих компонентами структурного тензора второго рода преобразованной $Q \mathscr{S}$ структуры, причем

$$
\begin{gathered}
\widetilde{A}_{[b c]}^{a d}=0, \\
\widetilde{A}_{b c}^{[a d]}=0 .
\end{gathered}
$$

Здесь мы воспользовались тем, что с учетом (21), (38) и (48)

$$
d B_{b}^{a}+B_{c}^{a} \theta_{b}^{c}-B_{b}^{c} \theta_{c}^{a}=0 .
$$

С другой стороны, в силу (49)

$$
d \theta_{b}^{a}=\theta_{c}^{a} \wedge \theta_{b}^{c}+\left(A_{b c}^{a h}+2 \sqrt{-1} \delta_{b}^{a} B_{c}^{h}\right) C_{h}^{d} \theta^{c} \wedge \theta_{d}
$$

Сравнивая это соотношение с (52), с учетом линейной независимости базисных форм находим, что

$$
\widetilde{A}_{b c}^{a d}=A_{b c}^{a h} C_{h}^{d} .
$$

ОПРЕДЕЛЕНИЕ 5. Почти контактное метрическое многообразие $M$ назьвается многообразием точечно постоянной $\Phi$-голоморфной секционной кривизнь с, если

$$
\langle R(X, \Phi X) X, \Phi X\rangle=c\|X\|^{4}, \quad X \in \mathscr{L}, \quad c \in C^{\infty}(M) .
$$

Еслик тому же $c=$ const, многообразие назьвается многообразием глобально постоянной $\Phi$-голоморфной секиионной кривизны. Многообразие Сасаки точечно постоянной $\Phi$-голоморфной секционной кривизны называется сасакиевой пространственной формой [14].

Пусть $M$ - почти контактное метрическое многообразие точечно постоянной $\Phi$-голоморфной секционной кривизны $c$. Соотношение (55) на пространстве присоединенной $G$-структуры запишется в форме

$$
4 R_{\widehat{a} b f \widehat{d}^{\widehat{a}}} X^{b} X^{f} X^{\widehat{d}}=-4 c g_{\widehat{a} b} g_{f \widehat{d}^{\widehat{a}}} X^{b} X^{f} X^{\widehat{d}}
$$

С учетом (1) это соотношение можно переписать в виде

$$
\left(R_{\widehat{a} b f \widehat{d}}+c \delta_{b}^{a} \delta_{f}^{d}\right) X^{\widehat{a}} X^{b} X^{f} X^{\widehat{d}}=0 .
$$

Поляризация этого соотношения приводит нас к следуюшему результату. 
ПРЕДЛОЖЕНИЕ 12. Почти контактное метрическое многообразие является многообразием точечно постоянной $\Phi$-голоморфной секиионной кривизныц с тогда и только тогда, когда

$$
R_{(b f)}^{(a)}=-\frac{c}{2} \widetilde{\delta}_{b f}^{a d}
$$

əде $\widetilde{\delta}_{b f}^{a d}=\delta_{b}^{a} \delta_{f}^{d}+\delta_{f}^{a} \delta_{b}^{d}$.

Пусть, в частности, $M-Q \mathscr{S}$-многообразие. Тогда с учетом $\left(24_{1}\right)$ и $(56)$ получаем

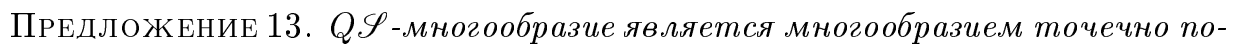
стоянной Ф-голоморфной секичонной кривизны тогда и только тогда, когда на пространстве присоединенной $G$-структурь

$$
A_{b c}^{a d}=3 B_{(b}^{(a} B_{c)}^{d)}-\frac{c}{2} \widetilde{\delta}_{b c}^{a d}
$$

əəe $\widetilde{\delta}_{b c}^{a d}=\delta_{b}^{a} \delta_{c}^{d}+\delta_{c}^{a} \delta_{b}^{d}$.

Следующий результат является контактным аналогом классической теоремы Шура для $Q \mathscr{S}$-многообразий класса $C R_{1}$.

Теорема 7. Точечное постоянство Ф-голоморфной секционной кривизны $Q \mathscr{S}$-многообразия класса $C R_{1}$ размерности свыше трех равносильно глобальному постоянству его $\Phi$-голоморфной секционной кривизны.

ДокАЗАтЕльство. Прежде всего отметим, что вторая группа структурных уравнений (19) $Q \mathscr{S}$-многообразия в случае $Q \mathscr{S}$-многообразий класса $C R_{1}$ с учетом (38) примет вид

$$
d \omega_{b}^{a}=\omega_{c}^{a} \wedge \omega_{b}^{c}+\left(A_{b c}^{a d}-2 B_{b}^{a} B_{c}^{d}\right) \omega^{c} \wedge \omega_{d}
$$

Стандартная процедура дифференциального продолжения этих соотношений с учетом (38) приводит к тождеству

$$
d A_{b c}^{a d}+A_{h c}^{a d} \omega_{b}^{h}+A_{b h}^{a d} \omega_{c}^{h}-A_{b c}^{h d} \omega_{h}^{a}-A_{b c}^{a h} \omega_{h}^{d}=A_{b c h}^{a d} \omega^{h}+A_{b c}^{a d h} \omega_{h},
$$

где $\left\{A_{b c h}^{a d}, A_{b c}^{a d h}\right\}$ - семейство функций на пространстве присоединенной $G$-структуры, симметричное по любой паре верхних либо нижних индексов. Продифференцируем с учетом (58), (21) и (38) соотношение (57):

$$
\frac{1}{2} \widetilde{\delta}_{b c}^{a d} d c=A_{b c h}^{a d} \omega^{h}+A_{b c}^{a d h} \omega_{h}
$$

Пусть $d c=c_{h} \omega^{h}+c^{h} \omega_{h}$. Сравнивая с предыдущим соотношением, с учетом линейной независимости базисных форм получаем, что

$$
\widetilde{\delta}_{b c}^{a d} c_{h}=2 A_{b c h}^{a d}
$$

Поскольку $A_{b c h}^{a d}=A_{b h c}^{a d}$, имеем отсюда, что $\widetilde{\delta}_{b c}^{a d} c_{h}=\widetilde{\delta}_{b h}^{a d} c_{c}$. Свертывая это соотношение сначала по индексам $c$ и $d$, а затем по индексам $a$ и $b$, получаем, что $\left(n^{2}-1\right) c_{h}=0$, а значит, $c_{h}=0, c^{h}=\bar{c}_{h}=0$. Следовательно, $d c=0$, т.е. $c=$ const. 
ЗАмечАнИЕ 5. Очевидно, любое трехмерное почти контактное метрическое многообразие является многообразием точечно постоянной, но, вообще говоря, не глобально постоянной $\Phi$-голоморфной секционной кривизны.

Tеорема 8. Пусть $M-Q \mathscr{S}$-многообразие класса $C R_{1}$ точечно постоянной Ф-голоморфной секиионной кривизны с. Тогда с точностью до ஜ̈-преобразования метрики либо $M$ - косимплектическое многообразие, либо $М$ является сасакиевой пространственной формой, либо $М$ локально эквивалентно произведению комплексного евклидова пространства на сасакиеву пространственную форму.

ДоКАЗАТЕЛЬСТво. Пусть эндоморфизм $B$ многообразия $M$ имеет единственное собственное значение $\lambda$. Если $\lambda=0$, то $\mathscr{B}=0$, а значит, $M$ - косимплектическое многообразие. В этом случае $\mathscr{B}$-преобразование метрики сводится к тождественному. Если $\lambda \neq 0$, то $B_{b}^{a}=\lambda \delta_{b}^{a}$, причем в силу косоэрмитовости оператора $B$ $\lambda$ - число чисто мнимое. В этом случае $C_{b}^{a}=-\frac{\sqrt{-1}}{\lambda} \delta_{b}^{a}$ и в силу (54) и (57)

$$
\begin{aligned}
\widetilde{A}_{b c}^{a d} & =A_{b c}^{a h} C_{h}^{d}=\left(3 B_{(b}^{(a} B_{c)}^{h)}-\frac{c}{2} \widetilde{\delta}_{b c}^{a h}\right) C_{h}^{d} \\
& =-\frac{3 \sqrt{-1}}{2}\left(B_{b}^{a} \delta_{c}^{d}+B_{c}^{a} \delta_{b}^{d}\right)-\frac{c}{2}\left(\delta_{b}^{a} C_{c}^{d}+\delta_{c}^{a} C_{b}^{d}\right) \\
& =\frac{\sqrt{-1}}{2}\left(\frac{c}{\lambda}-3 \lambda\right) \widetilde{\delta}_{b c}^{a d} .
\end{aligned}
$$

В силу предложений 13 и 2 сасакиева структура на $M$, полученная после $\mathscr{B}$-преобразования метрики, имеет постоянную $Ф$-голоморфную секционную кривизну.

Пусть теперь эндоморфизм $B$ имеет по крайней мере два различных собственных значения $\lambda$ и $\mu$. Обозначим $r_{1}=\operatorname{dim} \mathscr{D}_{\lambda}, r_{2}=\operatorname{dim} \mathscr{D}_{\mu}$. Построим сокрашенную $G$-структуру, состояшую из $A$-реперов, первые $r_{1}$ векторов которых принадлежат подпространству $\left(\mathscr{D}_{B}^{\lambda}\right)_{p}$, а следующие $r_{2}$ векторов - подпространству $\left(\mathscr{D}_{B}^{\mu}\right)_{p}$, где $p$ - вершина репера. Пусть индексы $\alpha, \beta, \gamma, \delta$ пробегают значения от 1 до $r_{1}$, а индексы $\varphi, \psi, \chi, \tau-$ значения от $r_{1}+1$ до $r_{1}+r_{2}$. Очевидно, на пространстве этой G-структуры

$$
B_{\beta}^{\alpha}=\lambda \delta_{\beta}^{\alpha}, \quad B_{\psi}^{\varphi}=\mu \delta_{\psi}^{\varphi}, \quad B_{\varphi}^{\alpha}=0, \quad B_{\alpha}^{\varphi}=0 .
$$

Дифференцируя последние два тождества, с учетом (21) и (38) получаем, что

$$
\omega_{\varphi}^{\alpha}=0, \quad \omega_{\alpha}^{\varphi}=0
$$

Дифференцируя эти соотношения внешним образом с учетом (19), убеждаемся, что на пространстве сокрашенной $G$-структуры

$$
A_{\varphi c}^{\alpha b}=0, \quad A_{\alpha c}^{\varphi b}=0 .
$$

Более того, соотношения (57) с учетом (60) и (61) на пространстве сокрашенной $G$-структуры примут вид:

$$
\begin{gathered}
A_{\gamma \delta}^{\alpha \beta}=3 B_{(\gamma}^{(\alpha} B_{\delta)}^{\beta)}-\frac{c}{2} \widetilde{\delta}_{\gamma \delta}^{\alpha \beta}, \quad A_{\psi \tau}^{\varphi \chi}=3 B_{(\psi}^{(\varphi} B_{\tau)}^{\chi)}-\frac{c}{2} \widetilde{\delta}_{\psi \tau}^{\varphi \chi} \\
0=A_{\beta \psi}^{\alpha \varphi}=\frac{3}{2} B_{\beta}^{\alpha} B_{\psi}^{\varphi}-\frac{c}{2} \delta_{\beta}^{\alpha} \delta_{\psi}^{\varphi} .
\end{gathered}
$$


С учетом (60) эти соотношения перепишутся в форме

$$
A_{\gamma \delta}^{\alpha \beta}=\frac{1}{2}\left(3 \lambda^{2}-c\right) \widetilde{\delta}_{\gamma \delta}^{\alpha \beta}, \quad A_{\psi \tau}^{\varphi \chi}=\frac{1}{2}\left(3 \mu^{2}-c\right) \widetilde{\delta}_{\psi \tau}^{\varphi \chi}, \quad 0=\frac{1}{2}(3 \lambda \mu-c) .
$$

В частности, из последнего соотношения следует, что эндоморфизм $B$ имеет не более двух (а значит, ровно два) различных собственных значений $\lambda$ и $\mu$, причем эти собственные значения связаны соотношением

$$
\lambda \mu=\frac{c}{3} .
$$

Если одно из этих собственных значений нулевое, из (62) следует, что $M$ - многообразие нулевой $\Phi$-голоморфной секционной кривизны. В этом случае в силу теоремы 4 многообразие $M$ локально эквивалентно произведению $Q \mathscr{S}$-многообразия, эндоморфизм $B$ которого имеет единственное (ненулевое) собственное значение, нулевой $\Phi$-голоморфной секционной кривизны на келерово многообразие нулевой голоморфной секционной кривизны, т.е. на многообразие $\mathbb{C}^{r_{2}}$. Как мы уже видели, первое из многообразий после $\mathscr{B}$-преобразования метрики является сасакиевой пространственной формой. Нетрудно подсчитать [6], что ее структура получается из стандартной сасакиевой структуры на нечетномерной сфере с помощью $D$-гомотетии с коэффициентом 4/3. Метрика же второго многообразия в процессе $\mathscr{B}$-преобразования согласно определению не меняется.

Пусть, наконец, $\lambda, \mu \neq 0$. Тогда $M$ - многообразие максимального ранга. С помощью $\mathscr{B}$-преобразования метрики оно переводится в многообразие Сасаки. Так же как и при выводе (59), находим из (62), что

$$
\begin{gathered}
\widetilde{A}_{\beta \gamma}^{\alpha \delta}=\frac{\sqrt{-1}}{2}\left(\frac{c}{\lambda}-3 \lambda\right) \widetilde{\delta}_{\beta \gamma}^{\alpha \delta}, \quad \widetilde{A}_{\chi \tau}^{\varphi \psi}=\frac{\sqrt{-1}}{2}\left(\frac{c}{\mu}-3 \mu\right) \widetilde{\delta}_{\chi \tau}^{\varphi \psi}, \\
\widetilde{A}_{\beta \psi}^{\alpha \varphi}=\frac{\sqrt{-1}}{2}\left(\frac{c}{\mu}-3 \lambda\right) \widetilde{\delta}_{\beta \psi}^{\alpha \varphi} .
\end{gathered}
$$

В силу (63) $c / \lambda-3 \lambda=c / \mu-3 \mu, c / \mu-3 \lambda=0$, а значит,

$$
\widetilde{A}_{b c}^{a d}=\frac{\sqrt{-1}}{2}\left(\frac{c}{\lambda}-3 \lambda\right) \widetilde{\delta}_{b c}^{a d}
$$

В силу предложений 13 и 2 сасакиева структура на $M$, полученная после $\mathscr{B}$-преобразования метрики, имеет постоянную $\Phi$-голоморфную секционную кривизну.

Как известно, косимплектическое многообразие локально эквивалентно произведению келерова многообразия на вещественную прямую [18]. В частности, косимплектическое многообразие постоянной $\Phi$-голоморфной секционной кривизны локально эквивалентно произведению комплексной пространственной формы (т.е. келерова многообразия постоянной голоморфной секционной кривизны) на вещественную прямую. С учетом хорошо известных классификаций комплексных пространственных форм [8] и сасакиевых пространственных форм [14], [6] из теоремы 8 получаем следующий результат, дающий полную классификацию (с точностью до $\mathscr{B}$-преобразования метрики) $Q \mathscr{S}$-многообразий класса $C R_{1}$ постоянной $\Phi$-голоморфной секционной кривизны. 


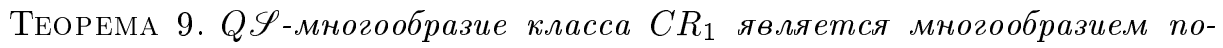
стоянной Ф-голоморфной секиионной кривизны тогда и только тогда, когда после $\mathscr{B}$-преобразования метрики оно локально әквивалентно одному из следующих многообразий:

1. произведению комплексного евклидова пространства на вещественную прямую;

2. произведению комплексного проективного пространства на вещественную прямую;

3. произведению комплексного гиперболического пространства на вещественную прямую;

4. произведению двумерного многообразия на вещественную прямую;

5. нечетномерной сфере, снабженной канонической сасакиевой структурой либо структурой, полученной из канонической с помощъю преобразования D-гомотетии;

6. нечетномерному аффинному пространству, снабженному канонической сасакиевой структурой постоянной $\Phi$-голоморфной секционной кривизны равной -3 ;

7. нечетномерной сфере, снабженной сасакиевой структурой, полученной из канонической с помощъю преобразования D-инверсии;

8. произведению многообразия Сасаки, полученного из нечетномерной сферь, снабженной стандартной сасакиевой структурой, с помощъю D-гомотетии с коэффициентом 4/3, на комплексное евклидово пространство.

ОПРЕДЕЛЕНИЕ 6 [16]. Говорят, что $(2 n+1)$-мерное почти контактное метрическое многообразие $M$ удовлетворяет аксиоме $\Phi$-голоморфных $(2 r+1)$-плоскостей, $1 \leqslant r \leqslant n$, если через каждую точку $p \in M$ для всякого $(2 r+1)$-мерного подпространства $L \subset T_{p}(M)$, инвариантного относительно действия структурного оператора $\Phi_{p}$, проходит $(2 r+1)$-мерное вполне геодезическое $\Phi$-инвариантное подмногообразие $N \subset M$ такое, что $T_{p}(N)=L$.

Пусть $M-(2 n+1)$-мерное $Q \mathscr{S}$-многообразие, удовлетворяющее аксиоме $\Phi$-голоморфных $(2 r+1)$-плоскостей, $p \in M, L \subset T_{p}(M)-(2 r+1)$-мерное $\Phi$-инвариантное подпространство, $N \subset M$ - соответствуюшее вполне геодезическое $\Phi$-инвариантное подмногообразие. В силу нечетномерности $N$ модуль $\mathfrak{X}(N)$ содержит ненулевой элемент ядра эндоморфизма $\left.\Phi\right|_{N}$, а значит, и векторное поле $\left.\xi\right|_{N}$, которое мы по-прежнему будем обозначать через $\xi$. Фиксируем $p \in N$. Пусть $\rho=\left(p, \beta_{0}=\xi_{p}, \beta_{1}, \ldots, \beta^{r}\right)-A$-репер комплексификации пространства $T_{p}(N)$. Если $j: N \rightarrow M, N \subset M,-$ естественное вложение, то, отождествляя векторы с их образами при отображении $\left(j_{*}\right)_{p}$ - диффференциале отображения $j$ в точке $p$, получаем в произвольном $A$-репере пространства $T_{p}(M)$ с учетом вешественности $j$

$$
\beta_{\alpha}=C_{\alpha}^{a} \varepsilon_{\alpha}, \quad \beta^{\alpha}=C_{a}^{\alpha} \varepsilon^{\alpha}, \quad \overline{C_{\alpha}^{a}}=C_{a}^{\alpha}
$$

Здесь и далее греческие индексы пробегают значения от 1 до $r$. Двойственные 
соотношения задаются уравнениями

$$
\begin{aligned}
\omega^{a} & =C_{\alpha}^{a} \theta^{\alpha} \\
\omega_{a} & =C_{a}^{\alpha} \theta_{\alpha} \\
\omega & =\theta
\end{aligned}
$$

где $\left(p, \theta, \theta^{1}, \ldots, \theta_{r}\right)$ - корепер, дуальный реперу $\rho$. Продифференцируем $\left(64_{1}\right)$ внешним образом с учетом (18):

$$
-C_{\alpha}^{a} d \theta^{\alpha}=\left(d C_{\alpha}^{a}-C_{\alpha}^{b} \omega_{b}^{a}-B_{b}^{a} C_{\alpha}^{b} \omega\right) \wedge \theta^{\alpha}
$$

Заметим, что $C_{\alpha}^{a} C_{a}^{\gamma}=\left\langle C_{\alpha}^{a} \varepsilon_{a}, C_{c}^{\gamma} \varepsilon_{c}\right\rangle=\left\langle\beta_{\alpha}, \beta^{\gamma}\right\rangle=\delta_{\alpha}^{\gamma}$. Поэтому, свертывая (65) с $C_{a}^{\gamma}$, получаем, что

$$
d \theta^{\gamma}=\theta_{\alpha}^{\gamma} \wedge \theta^{\alpha}+B_{\alpha}^{\gamma} \theta \wedge \theta^{\alpha}
$$

где $\theta_{\alpha}^{\gamma}=-C_{a}^{\gamma} d C_{\alpha}^{a}+C_{a}^{\gamma} C_{\alpha}^{b} \omega_{b}^{a}, B_{a}^{\gamma}=C_{\alpha}^{b} C_{\alpha}^{c} B_{b}^{a}$. Аналогично, дифференцируя соотношение $\left(64_{2}\right)$ внешним образом, получаем, что

$$
d \theta_{\gamma}=-\theta_{\gamma}^{\alpha} \wedge \theta_{\alpha}-B_{\gamma}^{\alpha} \theta \wedge \theta_{\alpha}
$$

При этом используется соотношение

$$
C_{a}^{\gamma} d C_{\alpha}^{a}+C_{\alpha}^{a} d C_{a}^{\gamma}=0
$$

полученное дифференцированием тождества $C_{\alpha}^{a} C_{a}^{\gamma}=\delta_{\alpha}^{\gamma}$. Далее, соотношение (65) с учетом (66) можно переписать в форме

$$
\left(d C_{\gamma}^{a}-C_{\gamma}^{b} \omega_{b}^{a}+C_{\alpha}^{a} \theta_{\gamma}^{\alpha}-\left(B_{b}^{a} C_{\gamma}^{b}-B_{\gamma}^{\alpha} C_{c}^{b}\right) \theta\right) \wedge \theta^{\gamma}=0
$$

откуда по лемме Картана находим, что

$$
d C_{\gamma}^{a}-C_{\gamma}^{b} \omega_{b}^{a}+C_{\alpha}^{a} \theta_{\gamma}^{\alpha}-\left(B_{b}^{a} C_{\gamma}^{b}-B_{\gamma}^{\alpha} C_{c}^{b}\right) \theta=C_{\gamma \beta}^{a} \theta^{\beta}
$$

где $C_{\beta \gamma}^{a}=C_{\gamma \beta}^{a}$ - система функций на пространстве присоединенной $G$-структуры, служаших компонентами второй квадратичной формы вложения $N \subset M$. Поскольку $N$ - вполне геодезическое подмногообразие, имеем $C_{\beta \gamma}^{a}=0$, а значит,

$$
d C_{\gamma}^{a}-C_{\gamma}^{b} \omega_{b}^{a}+C_{\alpha}^{a} \theta_{\gamma}^{\alpha}-\left(B_{b}^{a} C_{\gamma}^{b}-B_{\gamma}^{\alpha} C_{c}^{b}\right) \theta=0
$$

Наиболее удобно это выражение записывается в терминах формы первой канонической связности:

$$
d C_{\gamma}^{a}-C_{\gamma}^{b} \zeta_{b}^{a}+C_{\alpha}^{a} \eta_{\gamma}^{\alpha}=0
$$

где $\eta_{\gamma}^{\alpha}=\theta_{\gamma}^{\alpha}+B_{\gamma}^{\alpha} \theta$. Дифференцируя (68) внешним образом с учетом $\left(18^{\prime}\right)$ и (64), получаем:

$$
C_{\gamma}^{a} d \eta_{\alpha}^{\gamma}=C_{\delta}^{a} \eta_{\gamma}^{\delta} \wedge \eta_{\alpha}^{\gamma}+C_{\alpha}^{b} C_{\delta}^{c} C_{d}^{\varepsilon} A_{b c}^{a d} \theta^{\delta} \wedge \theta_{\varepsilon}
$$


С другой стороны, записывая (66) и (67) в виде

$$
d \theta^{\alpha}=\eta_{\gamma}^{\alpha} \wedge \theta^{\gamma}, \quad d \theta_{\alpha}=-\eta_{\alpha}^{\gamma} \wedge \theta_{\gamma}
$$

соответственно и находя дифференциальное продолжение этих соотношений, получаем, что

$$
d \eta_{\beta}^{\alpha}=\eta_{\gamma}^{\alpha} \wedge \eta_{\beta}^{\gamma}+\lambda_{\beta \gamma}^{\alpha \delta} \theta^{\gamma} \wedge \theta_{\delta}
$$

где $\left\{\lambda_{\beta \gamma}^{\alpha \delta}\right\}$ - система функций на пространстве соответствующей $G$-структуры, удовлетворяющая соотношениям $\lambda_{\beta \gamma}^{[\alpha \delta]}=\lambda_{[\beta \gamma]}^{\alpha \delta}=0$. Подставляя (70) в (69), с учетом линейной независимости базисных форм получаем

$$
C_{\alpha}^{a} \lambda_{\beta \gamma}^{\alpha \delta}=C_{\beta}^{b} C_{\gamma}^{c} C_{d}^{\delta} A_{b c}^{a d}
$$

Из единственности определения вполне геодезического подмногообразия по его начальным данным в какой-либо точке следует, что из выполнимости аксиомы $\Phi$-голоморфных $(2 r+1)$-плоскостей $(r \geqslant 1)$ на данном многообразии вытекает выполнимость на нем аксиомы 3 -плоскостей. Но тогда (71) принимает вид

$$
C^{a} \lambda=C^{b} C^{c} C_{d} A_{b c}^{a d}
$$

Из (72) видно, что $\lambda$ является однородной функцией первой степени по переменньп $C^{h}$. Продифференцируем (72) по этим переменным:

$$
\lambda \delta_{h}^{a}+C^{a} \frac{\partial \lambda}{\partial C^{h}}=A_{b c}^{a d} \delta_{h}^{b} C^{c} C_{d}+A_{b c}^{a d} C^{b} \delta_{h}^{c} C_{d}
$$

Свернем это соотношение по индексам $a$ и $h$. С учетом теоремы Эйлера об однородных функциях и соотношений (20) получим:

$$
\lambda=\frac{2}{n+1} A_{c}^{d} C^{c} C_{d}, \quad \text { где } A_{c}^{d}=A_{h c}^{h d} .
$$

Подставим это соотношение в (72):

$$
\left(A_{b c}^{a d}-\frac{2}{n+1} A_{c}^{d} \delta_{b}^{a}\right) C^{b} C^{c} C_{d}=0 .
$$

В силу произвола в выборе $C^{b}, C_{d}$ находим отсюда, что

$$
A_{b c}^{a d}=\frac{1}{n+1}\left(A_{b}^{a} \delta_{c}^{d}+A_{c}^{a} \delta_{b}^{d}\right) .
$$

Свернем это соотношение по индексам $a$ и $b: A_{c}^{d}=\frac{1}{n} A \delta_{c}^{a}$, где $A=A_{a}^{a}$, а значит,

$$
A_{b c}^{a d}=\frac{A}{n(n+1)} \widetilde{\delta}_{b c}^{a d} .
$$

Обратное очевидно: система Пфаффа, задающая $\Phi$-голоморфную $(2 r+1)$-плоскость, при выполнении соотношений (73) вполне интегрируема, а ее интегральные многообразия суть вполне геодезические подмногообразия. Таким образом, доказана 
ТЕОРЕМА 10. QS्S-многообразие удовлетворяет аксиоме Ф-голоморфных $(2 r+1)$-плоскостей тогда и только тогда, когда на пространстве присоединенной G-структуры компоненты структурного тензора второго рода удовлетворяют соотночениям (73).

Пусть теперь $M-Q \mathscr{S}$-многообразие точечно постоянной $\Phi$-голоморфной секционной кривизны $c$, удовлетворяюшее аксиоме $\Phi$-голоморфных $(2 r+1)$-плоскостей. В силу (57) и (73) на пространстве присоединенной $G$-структуры

$$
B_{b}^{a} B_{c}^{d}+B_{c}^{a} B_{b}^{d}=k \delta_{b c}^{a d}, \quad \text { где } \quad k=\frac{2 A}{3 n(n+1)}-\frac{c}{3}
$$

Как уже отмечалось, в силу косоэрмитовости оператора $B_{p}$ его матрица при подходящем выборе $A$-репера $\left\{p, \varepsilon_{a}, \varepsilon_{\widehat{b}}\right\}$ приводится к диагональному виду с чисто мнимьми собственными значениями, т.е.

$$
B_{b}^{a}=B_{b} \delta_{b}^{a}, \quad B_{b}=\sqrt{-1} \alpha_{b}, \quad \alpha_{b} \in \mathbb{R}, \quad b=1, \ldots, n
$$

Из (74) следует, что в таком репере $B_{b} B_{c}=k, b, c=1, \ldots, n$, следовательно, $\left(B_{b}-B_{c}\right)^{2}=0$, т.е. $B_{b}=B_{c}$, а значит, $B_{b}^{a}=\sqrt{-1} \alpha \delta_{b}^{a}$, где $\alpha-$ подходящая гладкая функция. Более того, из (21) и (38) вытекает, что $\alpha=$ const. Если $\alpha=0$, то $M-$ косимплектическое многообразие постоянной $\Phi$-голоморфной секционной кривизны. Если $\alpha \neq 0$, то $M$ - многообразие, гомотетичное сасакиевой пространственной форме, а значит, само является сасакиевой пространственной формой. Обратно, если $M$ - многообразие одного из перечисленных типов, то оно удовлетворяет аксиоме $\Phi$-голоморфиых $(2 r+1)$-плоскостей [5]. Тем самым доказана

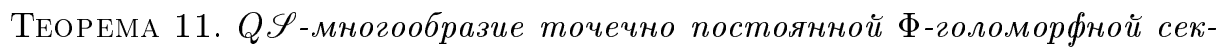
иионной кривизны удовлетворяет аксиоме $\Phi$-голоморфных $(2 r+1)$-плоскостей тогда и только тогда, когда оно либо является косимплектическим многообразием, либо гомотетично многообразию Сасаки. Полная классификация таких многообразий с точностью до гомотетии представлена многообразиями, перечисленными в пn. 1-7 теоремы 9.

СлЕДСТВИЕ. Многообразие, указанное в $п .8$ теоремы 9, будучи многообразием постоянной $\Phi$-голоморфной секиионной кривизны, не удовлетворяет аксиомам $\Phi$-голоморфных $(2 r+1)$-плоскостей.

Пусть, наконец, $M-Q \mathscr{S}$-многообразие класса $C R_{1}$, удовлетворяющее аксиоме $\Phi$-голоморфных $(2 r+1)$-плоскостей. В силу (73) и (54) для этого многообразия

$$
\widetilde{A}_{b c}^{a d}=\frac{A}{n(n+1)}\left(\delta_{b}^{a} C_{c}^{d}+\delta_{c}^{a} C_{b}^{d}\right)
$$

Если оператор $B$ многообразия нулевой, то $M$ - косимплектическое многообразие, которое в силу предложения 13 является многообразием постоянной $\Phi$-голоморфной секционной кривизны. 
Пусть $B$ - невырожденный эндоморфизм. В силу $\left(53_{2}\right)$ в случае $A \neq 0$ имеем:

$$
\delta_{b}^{a} C_{c}^{d}+\delta_{c}^{a} C_{b}^{d}=\delta_{b}^{d} C_{c}^{a}+\delta_{c}^{d} C_{b}^{a}
$$

Свертывая это соотношение по индексам $a$ и $b$, получаем, что

$$
C_{b}^{a}=\alpha \delta_{b}^{a}
$$

где $\alpha=\frac{1}{n} C_{h}^{h}$ - вешественная функция. Следовательно, $(\mathscr{B} \circ \Phi)^{-1}=\alpha \mathrm{id}$, откуда $\mathscr{B}=-\frac{1}{\alpha} \Phi, \alpha=$ const, т.е. $M$ - многообразие, гомотетичное многообразию Сасаки. В этом случае по предложению $13 M$ - многообразие постоянной $\Phi$-голоморфной секционной кривизны.

Пусть теперь $A=0$. Тогда $A_{b c}^{a d}=0$ и ввиду (54) $\widetilde{A}_{b c}^{a d}=0$. С учетом $\left(24_{1}\right)$ и предложения $2 \widetilde{R}_{\widehat{a} b c \widehat{d}}=-2 \delta_{b}^{a} \delta_{c}^{d}+\delta_{b}^{a} \delta_{c}^{d}$. Следовательно,

$$
\widetilde{R}_{(b c)}^{(a)}=\frac{3}{2} \widetilde{\delta}_{b c}^{a d}
$$

где $\widetilde{R}$ - тензор кривизны метрики $G$. По предложению $12 \mathscr{B}$-преобразованная структура является сасакиевой структурой постоянной $\Phi$-голоморфной секционной кривизны (равной -3$)$.

Пусть, наконец, $B$ - ненулевой, но вырожденный эндоморфизм. Построив, как и при доказательстве теоремы 8 , сокращенную $G$-структуру и проводя то же рассуждение, что и при выводе соотношения (62), получаем, что в этом случае $A=0$. Следовательно,

$$
A_{b c}^{a d}=0, \quad \text { в частности } A_{\beta \gamma}^{\alpha \delta}=0, \quad A_{\psi \tau}^{\varphi \chi}=0 .
$$

Напомним, что по теореме 4 многообразие $M$ в этом случае локально эквивалентно произведению $Q \mathscr{S}$-многообразия максимального ранга на келерово многообразие. Первое из них в силу (75) и доказанного выше $\mathscr{B}$-эквивалентно сасакиевой пространственной форме $\Phi$-голоморфной секционной кривизны (равной -3 ), а второе является плоским келеровым многообразием, а значит, локально эквивалентно комплексному евклидову пространству $\mathbb{C}^{r_{2}}$.

Обратно, очевидно, для любого из перечисленных типов многообразий выполняется тождество (73), а значит, оно удовлетворяет аксиоме $\Phi$-голоморфных $(2 r+1)$-плоскостей.

С учетом тех же соображений, которыми завершено доказательство теоремы 9 , получаем следующий результат, дающий полную классификацию (с точностью до $\mathscr{B}$-преобразования метрики) $Q \mathscr{S}$-многообразий класса $C R_{1}$, удовлетворяющих аксиоме $\Phi$-голоморфных $(2 r+1)$-плоскостей.

ТЕОРема 12. QS $\mathscr{S}$-многообразие класса $C R_{1}$ удовлетворяет аксиоме $\Phi$-голоморфных $(2 r+1)$-плоскостей тогда и только тогда, когда после Я̈В-преобразования метрики оно локально әквивалентно одному из следующих многообразий:

1. произведению комплексного евклидова пространства на вещественную пряммую; 
2. произведению комплексного проективного пространства на вещественную прямую;

3. произведению комплексного гиперболического пространства на вещественную прямую;

4. произведению двумерного многообразия на вещественную прямую;

5. нечетномерной сфере, снабженной канонической сасакиевой структурой либо структурой, полученной из канонической с помощъю преобразования D-гомотетии;

6. нечетномерному аффинному пространству, снабженному канонической сасакиевой структурой постоянной Ф-голоморфной секционной кривизны равной -3 ;

7. нечетномерной сфере, снабжснной сасакиевой структурой, полученной из канонической с помощью преобразования D-инверсии;

8. произведению многообразия вида (6) на комплексное евклидово пространство.

С учетом теорем 9 и 12 получаем

СлЕДСТВИЕ. Многообразие, указанное в $п .8$ теоремы 12, будучи многообразием, удовлетворяющим аксиоме $\Phi$-голоморфных $(2 r+1)$-плоскостей, не является многообразием постоянной Ф-голоморфной секционной кривизнъ.

Этот результат, как и следствие к теореме 11, наглядно демонстрирует отличие геометрии $Q \mathscr{S}$-многообразий от геометрии многообразий Сасаки, для которых вьполнимость указанной аксиомы равносильна постоянству $\Phi$-голоморфной секционной кривизны многообразия (см. [16]).

\section{Список литературы}

1. Chern S.-S. Pseudogroupes continus infinis // Colloques Internat. Centre Nat. Rech. Sci. 1953. V. 52. P. 119-136.

2. Gray J. W. Some global properties of contact structures // Ann. of Math. (2). 1959. V. 69. № 2. P. 421-450.

3. Sasaki $S$. On differentiable manifolds with certain structures which are closely related to almost contact structure. I // Tôhoku Math. J. (2). 1960. V. 12. № 3. P. 459-476.

4. Blair D. E. Contact manifolds in Riemannian geometry. Berlin: Springer-Verlag, 1976. (Lecture Notes in Math. V. 509.)

5. Кириченко B. $\Phi$. Методы обобщенной эрмитовой геометрии в теории почти контактных многообразий // Итоги науки и техн. Проблемы геометрии. Т. 18. М.: ВИНИТИ, 1986. C. $25-71$.

6. Кириченко В. $\Phi$. Аксиома $\Phi$-голоморфных плоскостей в контактной метрической геометрии // Изв. АН СССР. Сер. матем. 1984. Т. 48. № 4. С. 711-739.

7. Kirichenko V.F. Generalized quasi-Kaehlerian manifolds and axioms of $C R$-submanifolds in generalized Hermitian geometry. I // Geom. Dedicata. 1994. V. 51. P. 75-104.

8. Kirichenko V.F. Generalized quasi-Kaehlerian manifolds and axioms of $C R$-submanifolds in generalized Hermitian geometry. II // Geom. Dedicata. 1994. V. 52. P. 53-85.

9. Blair D. E. The theory of quasi-Sasakian structures // J. Differential Geom. 1967. V. 1. P. 331-345.

10. Tanno S. Quasi-Sasakian structures of rank $2 p+1 / /$ J. Differential Geom. 1971. V. 5 . P. 317-324.

11. Kanemaki Sh. Quasi-Sasakian manifolds // Tôhoku Math. J. (2). 1977. V. 29. P. 227-233. 
12. Yanamoto H. Quasi-Sasakian hypersurfaces in almost Hermitian manifolds // Res. Rep. Nagaoka Tech. College. 1969. V. 5. № 2. P. 149-158.

13. Kanemaki Sh. Products of $f$-manifolds // TRU Math. 1974. V. 10. P. 11-17.

14. Tanno S. Sasakian manifolds with constant $\varphi$-holomorphic sectional curvature // Tôhoku Math. J. (2). 1969. V. 21. P. 501-507.

15. Ogiue $K$. On almost contact manifolds admitting axiom of planes and axioms of free mobility // Kodai Math. Semin. Rep. 1964. V. 16. P. 223-232.

16. Ishihara I. Anti-invariant submanifolds of a Sasakian space forms // Kodai Math. J. 1979. V. 2. P. 171-186.

17. Кобаяии Ш., Номидзу К. Основы дифференциальной геометрии. М.: Наука, 1981.

18. Kiritchenko V.F. Sur le géométrie des variétés approximativement cosymplectiques // C. R. Acad. Sci. Paris. Sér. I. Math. 1982. V. 295. P. 673-676.

19. Kobayashi $S$. Principal fibre bundles with the 1-dimensional toroidal group // Tôhoku Math. J. (2). 1956. V. 8. P. 29-45.

Московский педагогический

Поступила в редакцию государственный университет 04.05 .2000 и 19.12 .2001 